\title{
Further Results on Diagonally Invariant Exponential Stability of Switching Linear Systems
}

\author{
Mihaela-Hanako Matcovschi $(\mathbb{D})$ and Octavian Pastravanu $(\mathbb{D}$ \\ Department of Automatic Control and Applied Informatics, "Gheorghe Asachi” Technical University of Iasi, 700050 Iasi, Romania \\ Correspondence should be addressed to Mihaela-Hanako Matcovschi; mhanako@ac.tuiasi.ro
}

Received 31 January 2018; Accepted 24 May 2018; Published 25 July 2018

Academic Editor: Francesco Cannizzaro

Copyright (C) 2018 Mihaela-Hanako Matcovschi and Octavian Pastravanu. This is an open access article distributed under the Creative Commons Attribution License, which permits unrestricted use, distribution, and reproduction in any medium, provided the original work is properly cited.

\begin{abstract}
The concept of diagonally invariant exponential stability (DIES) was originally introduced for single-model linear systems and subsequently expanded in the study of linear systems with interval-type uncertainties and linear systems with arbitrary switching. The results presented in this article refer to new approaches to DIES characterization for arbitrary switching systems, which exploit mathematical tools completely different from earlier work. The previous papers are based on the properties of matrix norms and measures applied to the constituent matrices defining the switching system, while the present paper uses the eigenvalues and eigenvectors of the column and row representatives built for a set of matrices derived from the constituent matrices of the switching system. The applicability of previous and new results, respectively, is illustrated by case studies (in both continuous- and discretetime) that lead to relevant comparisons between the two classes of analysis methods.
\end{abstract}

\section{Introduction}

1.1. Research Framework. The current paper considers the dynamics of switching linear systems described in discretetime by

$$
\begin{aligned}
\mathbf{x}(t+1) & =\mathbf{A}_{v(t)} \mathbf{x}(t), \\
\mathbf{x}\left(t_{0}\right) & =\mathbf{x}_{0},
\end{aligned}
$$

$$
\mathbf{A}_{v(t)} \in \mathscr{A}, t, t_{0} \in \mathbb{Z}_{+}, t \geq t_{0},
$$

and in continuous-time by

$$
\begin{aligned}
\dot{\mathbf{x}}(t) & =\mathbf{A}_{v(t)} \mathbf{x}(t), \\
\mathbf{x}\left(t_{0}\right) & =\mathbf{x}_{0},
\end{aligned}
$$

$$
\mathbf{A}_{v(t)} \in \mathscr{A}, t, t_{0} \in \mathbb{R}_{+}, t \geq t_{0},
$$

where

$$
\mathscr{A}=\left\{\mathbf{A}_{1}, \mathbf{A}_{2}, \ldots, \mathbf{A}_{N}\right\} \subset \mathbb{R}^{n \times n}
$$

is a set of matrices characterizing the $N$ linear modes exhibited by the switching system, and $v: \mathbb{Z}_{+} \longrightarrow\{1, \ldots, N\}$ (discrete-time) and, respectively, $v: \mathbb{R}_{+} \longrightarrow\{1,2, \ldots, N\}$ (continuous-time) are, respectively, an arbitrarily switching signal. At any time instant $t \in \mathbb{Z}_{+}$(discrete-time) or $t \in \mathbb{R}_{+}$ (continuous-time), the active mode of the switching system is defined by the subscript $\theta=v(t)$ associated with the matrix $\mathbf{A}_{\theta} \in \mathscr{A}$ that, respectively, uniquely generates the dynamics

$$
\mathbf{x}(t+1)=\mathbf{A}_{\theta} \mathbf{x}(t), \quad t \in \mathbb{Z}_{+}, \theta \in\{1, \ldots, N\}
$$

and

$$
\dot{\mathbf{x}}(t)=\mathbf{A}_{\theta} \mathbf{x}(t), \quad t \in \mathbb{R}_{+}, \theta \in\{1, \ldots, N\} .
$$

A system trajectory that starts from the initial condition $\mathbf{x}\left(t_{0}\right)=\mathbf{x}_{0} \in \mathbb{R}^{n}$ depends on the switching signal $v(\cdot)$, reason for which this dependence is outlined by the notation $\mathbf{x}(t)=$ $\mathbf{x}\left(t ; t_{0}, \mathbf{x}_{0}, v\right)$.

The continuous-time operation of the switching system $(1-\mathrm{H})$ is assumed to preserve the values of the state-space variables at any commutation time $\tau \in \mathbb{R}_{+}$; i.e., the equality $\lim _{h \downarrow 0} \mathbf{x}(\tau-h)=\mathbf{x}(\tau)$ is satisfied.

The notations " $\mathrm{S}$ " and " $\mathrm{H}$ " used above, as extension in equations numbering, come from the stability analysis intended by our work, which is addressed in the sense 
of Schur (abbreviated "S") for discrete-time dynamics and in the sense of Hurwitz (abbreviated " $H$ ") for continuoustime dynamics. The meaning of this notations will also be preserved when used as superscripts for some matrices.

Throughout the text the notation " $\mathrm{X} / / \mathrm{Y}$ " is used in place of " $\mathrm{X}$ " and, respectively, " $\mathrm{Y}$ " in order to organize a parallel presentation of the statements referring to analogous properties exhibited by discrete- and continuous-time systems.

The study of stability and stabilizability of switching and switched systems constituted the research interests of numerous control engineering groups during the past fifteen years. The monograph [1] presents the background existing in the early 2000, created by the pioneering works in this field. Later on, the picture is enlarged by the survey paper [2] and the monographs $[3,4]$, as well as by the numerous works cited therein. The recent period, meaning results reported after 2010, can be globally characterized as focusing on specialized classes of dynamics (such as dynamics with dwell time, e.g., [5-7]; delayed dynamics, e.g., [7-9]; positive dynamics, e.g., [10-12] - to mention just a few of the representative publications of the period we are referring to).

The nomenclature used by most of the cited works considers that the commutations of a switching system are determined by external signals, whereas the commutations of a switched system are controlled by internal signals. In accordance with this terminology, system $(3-\mathrm{S}) / /(3-\mathrm{H}) \mathrm{de}-$ scribed above exhibits an arbitrary switching, where the commutations between modes are driven by the signal $v$ : $\mathbb{Z}_{+} \longrightarrow\{1, \ldots, N\}$ (discrete-time), $v: \mathbb{R}_{+} \longrightarrow\{1,2, \ldots, N\}$ (continuous-time).

The concept of diagonally invariant exponential stability (DIES) to be analyzed by the current article was introduced by paper [13] for linear time-invariant systems whose discreteand continuous-time dynamics was described by a unique matrix. Paper [13] shows that DIES characterizes a particular type of exponential stability, reason for which the test instruments rely on algebraic properties stronger than the eigenvalue location, namely, inequalities involving matrix norms and matrix measures. The DIES concept was extended to interval systems by [14] and to switching systems of form (1-S)//(1-H) by [15] for discrete-time and [16] for continuoustime.

1.2. Research Objectives and Exposition Plan. The current research is founded on the DIES concept and its characterization, by equivalence, presented in our previous works [15] for discrete-time and [16] for continuous-time. The developments proposed in this article refer to novel approaches to DIES characterization that exploit mathematical instruments completely different from $[15,16]$.

Briefly speaking, the old results, corresponding to $[15,16]$, rely on the properties of matrix norms and matrix measures applied to the matrices $\mathbf{A}_{\theta} \in \mathbb{R}^{n \times n}, \theta=1, \ldots, N$, defining $\mathscr{A}(2)$, whereas the new results (herein developed) explore the spectra of the column and row representatives built for the matrix set $\mathscr{A}(2)$. It is worth mentioning that the new results have been derived only for the DIES analysis with respect to the Hölder $p$-norms, with $p \in\{1,2, \infty\}$, although the DIES concept is defined for $1 \leq p \leq \infty$. This fact should not be seen as a limitation of the new results, since most of the concrete problems are formulated relatively to the above-mentioned norms.

The exposition plan for the remainder of the text takes into consideration a short visit of the already existing results (also called old results) that are summarized by Section 2, by using a presentation style that unifies the discrete- and continuous-time cases (separately addressed by $[15,16]$, respectively). Section 3 develops the novel results in full accordance with the background created by the previous section and by using the same unified presentation style. Section 4 illustrates the applicability of the old and new results in parallel for concrete numerical examples and carefully compares similarities and differences between the two types of results. Some concluding remarks on the role and importance of our new results are formulated in Section 5.

The notations used throughout the whole text are presented by the appendix accompanying this article.

\section{Background: Results Available on DIES}

2.1. Definition and Characterization of DIES. The DIES concept was introduced by paper [13] for linear time-invariant systems whose discrete- and continuous-time dynamics are described by a unique matrix. Papers $[15,16]$ extended the DIES concept to switching linear systems in accordance with the following definition.

Definition 1. Let $1 \leq p \leq \infty, \mathbf{D}=\operatorname{diag}\left\{d_{1}, \ldots, d_{n}\right\}, d_{i}>0$, $i=1, \ldots, n$, and $0<r<1 / / r<0$. The switching linear system $(1-\mathrm{S}) / /(1-\mathrm{H})$ is called diagonally invariant exponentially stable relative to the p-norm and parameters $\mathrm{D}, r$ (abbreviated as $\operatorname{DIES}_{p}^{\mathbf{D}, r}$ ) under arbitrary switching if

$$
\begin{aligned}
& \forall \varepsilon>0, \\
& \forall t_{0} \in \mathbb{Z}_{+}, \\
& \forall \mathbf{x}_{0}=\mathbf{x}\left(t_{0}\right) \in \mathbb{R}^{n}, \\
& \forall v: \mathbb{Z}_{+} \longrightarrow\{1,2, \ldots, N\}: \\
&\left\|\mathbf{x}_{0}\right\|_{p}^{\mathbf{D}} \leq \varepsilon \Longrightarrow\left\|\mathbf{x}\left(t ; t_{0}, \mathbf{x}_{0}, v\right)\right\|_{p}^{\mathbf{D}} \leq \varepsilon r^{t-t_{0}},
\end{aligned}
$$

$$
\forall t \in \mathbb{Z}_{+}, t \geq t_{0}
$$

and, respectively,

$$
\begin{aligned}
& \forall \varepsilon>0, \\
& \forall t_{0} \in \mathbb{R}_{+}, \\
& \forall \mathbf{x}_{0}=\mathbf{x}\left(t_{0}\right) \in \mathbb{R}^{n}, \\
& \forall v: \mathbb{R}_{+} \longrightarrow\{1,2, \ldots, N\}: \\
&\left\|\mathbf{x}_{0}\right\|_{p}^{\mathbf{D}} \leq \varepsilon \longrightarrow\left\|\mathbf{x}\left(t ; t_{0}, \mathbf{x}_{0}, v\right)\right\|_{p}^{\mathbf{D}} \leq \varepsilon e^{r\left(t-t_{0}\right)}, \\
& \forall t \in \mathbb{R}_{+}, t \geq t_{0} .
\end{aligned}
$$

In (4-S) and (4-H), the vector norm $\|\mathbf{x}\|_{p}^{\mathbf{D}}=\left\|\mathbf{D}^{-1} \mathbf{x}\right\|_{p}$ is used in accordance with the details given in the appendix. 
A qualitative analysis developed in terms of invariant sets reveals that DIES $S_{p}^{\mathrm{D}, r}$ property expresses the invariance of the exponentially decreasing time-dependent sets (or, equivalently, contractive sets)

$$
\begin{aligned}
X_{p}^{\mathbf{D}, r}\left(\varepsilon ; t, t_{0}\right)=\left\{\mathbf{x} \in \mathbb{R}^{n} \mid\|\mathbf{x}\|_{p}^{\mathbf{D}}\right. & \left.\leq \varepsilon r^{t-t_{0}}\right\}, \\
t, t_{0} & \in \mathbb{Z}_{+}, t \geq t_{0}, \varepsilon>0,
\end{aligned}
$$

and, respectively,

$$
\begin{aligned}
X_{p}^{\mathbf{D}, r}\left(\varepsilon ; t, t_{0}\right)=\left\{\mathbf{x} \in \mathbb{R}^{n} \mid\|\mathbf{x}\|_{p}^{\mathbf{D}}\right. & \left.\leq \varepsilon e^{r\left(t-t_{0}\right)}\right\}, \\
t, t_{0} & \in \mathbb{R}_{+}, t \geq t_{0}, \varepsilon>0,
\end{aligned}
$$

with respect to the trajectories of the switching linear system $(1-\mathrm{S}) / /(1-\mathrm{H})$ for any switching sequence $v$.

For further details on exploring the connection between set invariance and the standard definition of (local and global) exponential stability, the reader is referred to the discussions in paper [13]. For instance, the $\operatorname{DIES}_{p}^{\mathbf{D}, r}$ concept implies the satisfaction of the classical $\delta-\varepsilon$ definition of the exponential stability of the equilibrium $\{0\}$ of system (1-S)// $(1-\mathrm{H})$, by using the vector norm \|\|$_{p}^{\mathbf{D}}$ and the precise value $\delta(\varepsilon)=\varepsilon$ for satisfying the inequality $\delta(\varepsilon) \geq \varepsilon$. In other words, the equilibrium $\{0\}$ may be exponentially stable, but the switching system $(1-\mathrm{S}) / /(1-\mathrm{H})$ does not have invariant sets of form $(5-\mathrm{S}) / /(5-\mathrm{H})$.

In relation $(5-\mathrm{S}) / /(5-\mathrm{H})$ the diagonal entries of matrix $\mathbf{D}$ scale the state variables, and the constant $0<r<1 / / r<0$ represents the contraction rate of the considered time-dependent sets. For the usual $p$-norms defined by $p \in\{1,2, \infty\}$, the contractive sets $(5-\mathrm{S}) / /(5-\mathrm{H})$ have well-known geometric shapes: hyper-rhombus, ellipses, and rectangles, respectively.

The DIES $S_{p}^{\mathrm{D}, r}$ property of switching linear systems of form $(1-\mathrm{S}) / /(1-\mathrm{H})$ is characterized by the following theorem that employs the matrix norms and measures defined in the appendix. This theorem merges, in a unified form, the results proven in [15] for the discrete-time case and in [16] for the continuous-time case.

Theorem 2. Let $1 \leq p \leq \infty, \mathbf{D}>0$ diagonal, and $0<r<$ $1 / / r<0$. The following statements are equivalent:

(i) The switching system (1-S)//(1-H) is DIES $S_{p}^{\mathrm{D}, r}$ under arbitrary switching.

(ii) The function

$$
\begin{gathered}
\mathscr{V}: \mathbb{R}^{n} \longrightarrow \mathbb{R}_{+}, \\
\mathscr{V}(\mathbf{x})=\|\mathbf{x}\|_{p}^{\mathbf{D}}
\end{gathered}
$$

is a strong Lyapunov function for the switching system $(1-\mathrm{S}) / /(1-\mathrm{H})$, which exhibits the decreasing rate $r$ along each nontrivial trajectory of the considered system; i.e.,

$$
\forall t \in \mathbb{Z}_{+}: \mathscr{V}(\mathbf{x}(t+1)) \leq r \mathscr{V}(\mathbf{x}(t))
$$

and, respectively,

$$
\begin{aligned}
\forall t & \in \mathbb{R}_{+}: D_{t}^{+} \mathscr{V}(\mathbf{x}(t)) \\
& =\lim _{h \downarrow 0} \frac{\mathscr{V}(\mathbf{x}(t+h))-\mathscr{V}(\mathbf{x}(t))}{h} \leq r \mathscr{V}(\mathbf{x}(t)) .
\end{aligned}
$$

(iii) The matrices $\mathbf{A}_{\theta}, \theta=1, \ldots, N$, defining the component subsystems of the switching system $(1-\mathrm{S}) / /(1-\mathrm{H})$ satisfy the inequalities

$$
\left\|\mathbf{A}_{\theta}\right\|_{p}^{\mathbf{D}} \leq r, \quad \theta=1, \ldots, N,
$$

and, respectively,

$$
\mu_{p}^{\mathrm{D}}\left(\mathbf{A}_{\theta}\right) \leq r, \quad \theta=1, \ldots, N .
$$

Proof. See the proofs of Theorem 2 in [15] for the discretetime case and Theorem 1 in [16] for the continuous-time case.

Inequalities $(8-\mathrm{S}) / /(8-\mathrm{H})$ may be utilized in testing the DIES $_{p}^{\mathbf{D}, r}$ of system $(1-\mathrm{S}) / /(1-\mathrm{H})$. It is important to notice the necessary and sufficient role of these inequalities in the $\operatorname{DIES}_{p}^{\mathbf{D}, r}$ characterization, because other works, such as $[2,17]$, mention their use just as sufficient conditions for uniform asymptotic stability.

For a single-model system, the DIES characterization via $(8-\mathrm{S}) / /(8-\mathrm{H})$ operates in the particular form $\|\mathbf{A}\|_{p}^{\mathbf{D}} \leq r$ $/ / \mu_{p}^{\mathbf{D}}(\mathbf{A}) \leq r$ presented in our previous work [13]. This matrix-norm//matrix-measure inequality implies the Schur// Hurwitz property of matrix $\mathbf{A}$, a fact which offers a supplementary motivation for the discussion on "DIES stronger compared to exponential stability" developed above, after Definition 1. An interesting consequence refers to the dynamics of the arbitrary switching system $(1-\mathrm{S}) / /(1-\mathrm{H})$, analyzed from the perspective of the individual dynamics exhibited by the $N$ constituent subsystems. Thus, it is well known that system $(1-\mathrm{S}) / /(1-\mathrm{H})$ is not necessarily stable if all its constituent subsystems are exponentially stable. On the other hand, if there exist a matrix $\mathbf{D}>0$ diagonal and $N$ constants $0<r_{1}, \ldots, r_{N}<1 / / r_{1}, \ldots, r_{N}<0$, such that the $N$ constituent subsystems are DIES $S_{p}^{\mathbf{D}, r_{1}}, \ldots$, DIES $_{p}^{\mathbf{D}, r_{N}}$, respectively, then system $(1-\mathrm{S}) / /(1-\mathrm{H})$ is $\operatorname{DIES}_{p}^{\mathbf{D}, r}$, for $r=$ $\max \left\{r_{1}, \ldots, r_{N}\right\}$.

2.2. Particular Forms of Inequalities $(8-\mathrm{S}) / /(8-\mathrm{H})$ in Theorem 2 for $p \in\{1,2, \infty\}$. The easy to handle, concrete forms of the matrix norms//matrix measures corresponding to $p \in$ $\{1,2, \infty\}$ (see Appendix) allow a convenient reformulation of inequalities $(8-\mathrm{S}) / /(8-\mathrm{H})$ in Theorem 2 , as shown by Theorem 3 stated below, which joins results proven in [15] for the discrete-time case and in [16] for the continuous-time case.

Consider the set of matrices $\mathscr{A}=\left\{\mathbf{A}_{1}, \mathbf{A}_{2}, \ldots, \mathbf{A}_{N}\right\}$ (2) that generates the dynamics of switching system $(1-\mathrm{S}) / /(1-\mathrm{H})$. For the discrete-time case, define the set of matrices $\mathscr{A}^{S}=$ $\left\{\mathbf{A}_{1}^{S}, \mathbf{A}_{2}^{S}, \ldots, \mathbf{A}_{N}^{S}\right\}$, where

$$
\left[A_{\theta}^{S}\right]_{i j}=\left|\left[A_{\theta}\right]_{i j}\right|, \quad i, j=1, \ldots, n, \theta=1, \ldots, N,
$$


and for the continuous-time case, the set of matrices $\mathscr{A}^{H}=$ $\left\{\mathbf{A}_{1}^{H}, \mathbf{A}_{2}^{H}, \ldots, \mathbf{A}_{N}^{H}\right\}$, where

$$
\left[A_{\theta}^{H}\right]_{i j}=\left\{\begin{array}{l}
{\left[A_{\theta}\right]_{i j}} \\
\left|\left[A_{\theta}\right]_{i j}\right|
\end{array},\right.
$$

$$
i, j=1, \ldots, n, \theta=1, \ldots, N \text {. }
$$

Theorem 3. Let $\mathbf{D}=\operatorname{diag}\left\{d_{1}, \ldots, d_{n}\right\}, d_{i}>0, i=1, \ldots, n$, and $r \in \mathbb{R}$.

(i) For $p=1$, inequalities $(8-\mathrm{S}) / /(8-\mathrm{H})$ are equivalent to

$$
\mathbf{v}^{T} \mathbf{A}_{\theta}^{S} \leq r \mathbf{v}^{T}, \quad \theta=1, \ldots, N
$$

and

$$
\mathbf{v}^{T} \mathbf{A}_{\theta}^{H} \leq r \mathbf{v}^{T}, \quad \theta=1, \ldots, N
$$

respectively, where $\mathbf{v}=\left[1 / d_{1} \cdots 1 / d_{n}\right]^{T} \in \mathbb{R}^{n}$ is a positive vector formed with the inverses of the diagonal entries of matrix D.

(ii) For $p=2$, inequalities $(8-\mathrm{S}) / /(8-\mathrm{H})$ are equivalent to

$$
\mathbf{A}_{\theta}^{T} \mathbf{Q} \mathbf{A}_{\theta}-r^{2} \mathbf{Q} \preceq 0, \quad \theta=1, \ldots, N,
$$

and

$$
\left(\mathbf{A}_{\theta}\right)^{T} \mathbf{Q}+\mathbf{Q A}_{\theta}-2 r \mathbf{Q} \preceq 0, \quad \theta=1, \ldots, N
$$

respectively, where $\mathbf{Q}=\left(\mathbf{D}^{-1}\right)^{2}$ is a positive definite diagonal matrix, formed with the square values of the inverses of the diagonal entries of matrix $\mathbf{D}$.

(iii) For $p=\infty$, inequalities $(8-\mathrm{S}) / /(8-\mathrm{H})$ are equivalent to

$$
\mathbf{A}_{\theta}^{S} \mathbf{v} \leq r \mathbf{v}, \quad \theta=1, \ldots, N
$$

and

$$
\mathbf{A}_{\theta}^{H} \mathbf{v} \leq r \mathbf{v}, \quad \theta=1, \ldots, N
$$

respectively, where $\mathbf{v}=\left[d_{1} \cdots d_{n}\right]^{T} \in \mathbb{R}^{n}$ is a positive vector formed with the diagonal entries of matrix $\mathbf{D}$.

Proof. See the proofs of Corollary 1 in [15] for the discretetime case and Corollary 1 in [16] for the continuous-time case.

Papers $[15,16]$ recommend the use of the above results in the sense that, for a chosen (or given) value of $0<r<1 / / r<$ 0 , Theorem 3 permits the search for a diagonal matrix $\mathbf{D}>0$. Thus, inequalities $(10-\mathrm{S}) / /(10-\mathrm{H})$ and $(12-\mathrm{S}) / /(12-\mathrm{H})$ can be numerically approached as LP problems, and (11-S)//(11-H) as LMI problems.

It is worth noticing that $\operatorname{DIES}_{p}^{\mathrm{D}, r}$ testing in the manner of the concomitant search for the parameters $\mathrm{D}, r$ requires a different point of view on the numerical exploitation of Theorem 3. For instance, one can devise algorithms that involve the iterative use of the LP or LMI problems (providing matrix D), correlated to an optimization scheme looking for the value $r$, e.g., a bisection method [18]. Another numerical approach may regard inequalities $(10-\mathrm{S}) / /(10-\mathrm{H})$ to $(12-\mathrm{S}) / /(12-\mathrm{H})$ as BMI problems, in accordance with the key principles discussed by [19].

\section{New Results: Column and Row Representatives-Based Approach to DIES}

This section shows that the $\operatorname{DIES}_{p}^{\mathbf{D}, r}$ characterization, for $p \epsilon$ $\{1,2, \infty\}$, expressed by inequalities $(8-S) / /(8-H)$ in Theorem 2 can also be addressed in terms of eigenvalues of some special matrices. These matrices are constructed from the set $\mathscr{A}=$ $\left\{\mathbf{A}_{1}, \mathbf{A}_{2}, \ldots, \mathbf{A}_{N}\right\}$ that generates the dynamics of switching system $(1-\mathrm{S}) / /(1-\mathrm{H})$, relying on the concepts of column and row representatives of a matrix set.

The eigenvalue employment in the $\operatorname{DIES}_{p}^{\mathrm{D}, r}$ testing for switching linear systems represents an important (and, in principle, expected) generalization of the results reported in [13] for $\mathrm{DIES}_{p}^{\mathrm{D}, r}, p \in\{1,2, \infty\}$, of linear time-invariant systems (single mode systems).

3.1. Column and Row Representatives for a Set of Matrices as Analytical Tools. Let $\mathscr{M}=\left\{\mathbf{M}_{1}, \ldots, \mathbf{M}_{N}\right\} \subset \mathbb{R}^{n \times n}$ be a set of matrices. Given a function $\sigma:\{1, \ldots, n\} \rightarrow\{1, \ldots, N\}$, denote by $\sigma=(\sigma(1), \ldots, \sigma(n))$ the corresponding $n$-tuple and represent by $\mathscr{C}$ the set of all the $n$-tuples with elements from $\{1, \ldots, N\}$.

For any $\boldsymbol{\sigma} \in \mathscr{C}$, we construct the matrix $\underline{\mathbf{M}}_{\sigma} \in \mathbb{R}^{n \times n}$, whose first column is the first column of $\mathbf{M}_{\sigma(1)}$ (denoted as $\left.\left[\mathbf{M}_{\sigma(1)}\right]_{(:, 1)}\right)$, second column is the second column of $\mathbf{M}_{\sigma(2)}$ (denoted as $\left[\mathbf{M}_{\sigma(2)}\right]_{(:, 2)}$ ), and so on; that is, $\underline{\mathbf{M}}_{\sigma}=$ $\left[\left[\mathbf{M}_{\sigma(1)}\right]_{(:, 1)} \cdots\left[\mathbf{M}_{\sigma(n)}\right]_{(:, n)}\right] \in \mathbb{R}^{n \times n}$. A matrix $\underline{\mathbf{M}}_{\sigma} \in \mathbb{R}^{n \times n}$, $\boldsymbol{\sigma} \in \mathscr{C}$, constructed as above is called a column representative of the matrix set $\mathscr{M}$. We say that $\sigma \in \mathscr{C}$ labels the column representatives.

For any $\boldsymbol{\sigma} \in \mathscr{C}$, we construct the matrix $\overline{\mathbf{M}}_{\boldsymbol{\sigma}} \in$ $\mathbb{R}^{n \times n}$, whose first row is the first row of $\mathbf{M}_{\sigma(1)}$ (denoted as $\left.\left[\mathbf{M}_{\sigma(1)}\right]_{(1,:)}\right)$, second row is the second row of $\mathbf{M}_{\sigma(2)}$ (denoted as $\left.\left[\mathbf{M}_{\sigma(2)}\right]_{(2,:)}\right)$, and so on; that is, $\overline{\mathbf{M}}_{\boldsymbol{\sigma}}=\left[\begin{array}{c}{\left[\mathbf{M}_{\sigma(1)}\right]_{(1, ;)}} \\ {\left[\mathbf{M}_{\sigma(\mathrm{n})}\right]_{(n,:)}}\end{array}\right] \in \mathbb{R}^{n \times n} \cdot \mathbf{A}$ matrix $\overline{\mathbf{M}}_{\boldsymbol{\sigma}} \in \mathbb{R}^{n \times n}, \boldsymbol{\sigma} \in \mathscr{C}$, constructed as above is called a row representative of the matrix set $\mathscr{M}$. We say that $\sigma \in \mathscr{C}$ labels the row representatives.

If the matrices $\mathbf{M}_{\theta} \in \mathbb{R}^{n \times n}, \theta=1, \ldots, N$, are (essentially) nonnegative, then all column representatives $\underline{\mathbf{M}}_{\sigma} \in \mathbb{R}^{n \times n}$, $\boldsymbol{\sigma} \in \mathscr{C}$, and all row representatives $\overline{\mathbf{M}}_{\boldsymbol{\sigma}} \in \mathbb{R}^{n \times n}, \boldsymbol{\sigma} \in \mathscr{C}$, are (essentially) nonnegative matrices. Subsequently, relying on Perron-Frobenius theory, one can define

$$
\begin{aligned}
& \underline{\lambda}^{*}=\max _{\boldsymbol{\sigma} \in \mathscr{C}} \lambda_{\max }\left(\underline{\mathbf{M}}_{\boldsymbol{\sigma}}\right), \\
& \underline{\mathscr{C}}^{*}=\left\{\boldsymbol{\sigma}^{*} \in \mathscr{C} \mid \lambda_{\max }\left(\underline{\mathbf{M}}_{\boldsymbol{\sigma}^{*}}\right)=\underline{\lambda}^{*}\right\},
\end{aligned}
$$

where $\underline{\lambda}^{*}$ represents the dominant eigenvalue of the column representatives and $\underline{\mathscr{C}}^{*}$ the set of labels associated with $\underline{\lambda}^{*}$. Similarly, one can define

$$
\begin{aligned}
& \bar{\lambda}^{*}=\max _{\boldsymbol{\sigma} \in \mathscr{C}} \lambda_{\max }\left(\overline{\mathbf{M}}_{\boldsymbol{\sigma}}\right), \\
& \overline{\mathscr{C}}^{*}=\left\{\boldsymbol{\sigma}^{*} \in \mathscr{C} \mid \lambda_{\max }\left(\overline{\mathbf{M}}_{\boldsymbol{\sigma}^{*}}\right)=\bar{\lambda}^{*}\right\},
\end{aligned}
$$

where $\bar{\lambda}^{*}$ represents the dominant eigenvalue of the row representatives and $\overline{\mathscr{C}}^{*}$ the set of labels associated with $\bar{\lambda}^{*}$. 
In the next two subsections, regarding the switching system $(1-\mathrm{S}) / /(1-\mathrm{H})$ and $(2)$, we are going to exploit the following:

(a) For the discrete-time case, the representatives of the matrix set $\mathscr{A}^{S}=\left\{\mathbf{A}_{1}^{S}, \mathbf{A}_{2}^{S}, \ldots, \mathbf{A}_{N}^{S}\right\}$ are defined by (9-S), the dominant eigenvalue of the column representatives is defined by

$$
\begin{aligned}
\left(\underline{\lambda}^{S}\right)^{*} & =\max _{\boldsymbol{\sigma} \in \mathscr{C}} \lambda_{\max }\left(\underline{\mathbf{A}}_{\sigma}^{S}\right), \\
\underline{\mathscr{C}}^{S^{*}} & =\left\{\boldsymbol{\sigma}^{*} \in \mathscr{C} \mid \lambda_{\max }\left(\underline{\mathbf{A}}_{\sigma^{*}}^{S}\right)=\left(\underline{\lambda}^{S}\right)^{*}\right\},
\end{aligned}
$$

and the dominant eigenvalue of the row representatives is defined by

$$
\begin{aligned}
\left(\bar{\lambda}^{S}\right)^{*} & =\max _{\boldsymbol{\sigma} \in \mathscr{C}} \lambda_{\max }\left(\overline{\mathbf{A}}_{\boldsymbol{\sigma}}^{S}\right), \\
\overline{\mathscr{C}}^{S^{*}} & =\left\{\boldsymbol{\sigma}^{*} \in \mathscr{C} \mid \lambda_{\max }\left(\overline{\mathbf{A}}_{\boldsymbol{\sigma}^{*}}^{S}\right)=\left(\bar{\lambda}^{S}\right)^{*}\right\} .
\end{aligned}
$$

(b) For the continuous-time case, the representatives of the matrix set $\mathscr{A}^{H}=\left\{\mathbf{A}_{1}^{H}, \mathbf{A}_{2}^{H}, \ldots, \mathbf{A}_{N}^{H}\right\}$ are defined by $(9-\mathrm{H})$, the dominant eigenvalue of the column representatives is defined by

$$
\begin{aligned}
\left(\underline{\lambda}^{H}\right)^{*} & =\max _{\boldsymbol{\sigma} \in \mathscr{C}} \lambda_{\max }\left(\underline{\mathbf{A}}_{\sigma}^{H}\right), \\
\underline{\mathscr{C}}^{H^{*}} & =\left\{\boldsymbol{\sigma}^{*} \in \mathscr{C} \mid \lambda_{\max }\left(\underline{\mathbf{A}}_{\sigma^{*}}^{H}\right)=\left(\underline{\lambda}^{H}\right)^{*}\right\},
\end{aligned}
$$

and the dominant eigenvalue of the row representatives is defined by

$$
\begin{aligned}
\left(\bar{\lambda}^{H}\right)^{*} & =\max _{\boldsymbol{\sigma} \in \mathscr{C}} \lambda_{\max }\left(\overline{\mathbf{A}}_{\boldsymbol{\sigma}}^{H}\right), \\
\overline{\mathscr{C}}^{H^{*}} & =\left\{\boldsymbol{\sigma}^{*} \in \mathscr{C} \mid \lambda_{\max }\left(\overline{\mathbf{A}}_{\boldsymbol{\sigma}^{*}}^{H}\right)=\left(\bar{\lambda}^{H}\right)^{*}\right\} .
\end{aligned}
$$

\subsection{Necessary and Sufficient Conditions for DIES ${ }_{p}^{\mathrm{D}, r}$ with $p \in$ $\{1, \infty\}$}

Theorem 4. (a) There exist $\mathbf{D}>0$ diagonal and $0<r<1$ such that system (1-S) is DIES $S_{1}^{\mathbf{D}, r}$ under arbitrary switching, if and only if $0<\left(\underline{\lambda}^{S}\right)^{*}<1$.

(b) There exist $\mathbf{D}>0$ diagonal and $r<0$ such that system $(1-\mathrm{H})$ is DIES $S_{1}^{\mathbf{D}, r}$ under arbitrary switching, if and only if $\left(\underline{\lambda}^{H}\right)^{*}<0$.

Proof. We give the proof only for the continuous-time case (b); the proof of discrete-time case (a) may be addressed along the same main lines.

Theorem 2 shows that system $(1-\mathrm{H})$ is $\mathrm{DIES}_{1}^{\mathbf{D}, r}$ under arbitrary switching, if and only if inequalities $(8-\mathrm{H})$ are satisfied. On the other hand, in accordance with Theorem 3(i), inequalities $(8-\mathrm{H})$ are equivalent to inequalities $(10-\mathrm{H})$, and therefore the proof relies on the use of the latter.
Only If. We first show that if inequalities $(10-\mathrm{H})$ have solutions $\mathbf{v}>0, r \in \mathbb{R}$, then $r$ satisfies the inequality $r \geq\left(\underline{\lambda}^{H}\right)^{*}$. Indeed, assume that $r<\left(\underline{\lambda}^{H}\right)^{*}$ and $\mathbf{v}>0 ; r$ solves the inequality (inequalities) $\mathbf{v}^{T} \underline{\mathbf{A}}_{\sigma^{*}}^{\bar{H}} \leq r \mathbf{v}^{T}, \boldsymbol{\sigma}^{*} \in \underline{\mathscr{C}}^{H^{*}}$. By taking $r=\left(\underline{\lambda}^{H}\right)^{*}-\varepsilon, \varepsilon>0$ and referring to one of the matrices $\underline{\mathbf{A}}_{\sigma^{*}}^{H}$, we get the inequality $\left(\left(\underline{\lambda}^{H}\right)^{*} \mathbf{I}-\underline{\mathbf{A}}_{\sigma^{*}}^{H}\right)^{T} \mathbf{v} \geq \varepsilon \mathbf{v}$ with solutions $\mathbf{v}>0$. If we use the notation $\mathbf{M}_{\boldsymbol{\sigma}^{*}}=\left(\left(\underline{\lambda}^{H}\right)^{*} \mathbf{I}-\underline{\mathbf{A}}_{\sigma^{*}}^{H}\right)^{T}$, then $\mathbf{M}_{\boldsymbol{\sigma}^{*}}$ is an M-matrix, and, for all the nonzero components of the vector $\mathbf{v}>0$, we have $\left(\mathbf{M}_{\boldsymbol{\sigma}^{*} \mathbf{v}}\right)_{i} \geq \boldsymbol{\varepsilon} \mathbf{v}_{i}>0$. This contradicts Theorem 6.4.6 (A5) from ([20], p. 149). Thus, the conditions $r<0$ and $r \geq\left(\underline{\lambda}^{H}\right)^{*}$ imply the inequality $\left(\underline{\lambda}^{H}\right)^{*}<0$.

If. Define the matrices $\mathbf{M}_{\theta}=r \mathbf{I}-\mathbf{A}_{\theta}^{H}, \theta=1, \ldots, N$, with $\left(\underline{\lambda}^{H}\right)^{*} \leq r<0$. In accordance with Exercise 1.3 .7 ([20], p. 9), the following two algebraic systems are dual:

$$
(\mathrm{S})\left\{\begin{array}{l}
{\left[\begin{array}{c}
\left(\mathbf{M}_{1}\right)^{T} \\
\cdots \\
\left(\mathbf{M}_{N}\right)^{T}
\end{array}\right] \mathbf{v} \geq 0} \\
\mathbf{v}>0
\end{array}\right.
$$

and

$$
(\widetilde{\mathrm{S}})\left\{\begin{array}{c}
{\left[\begin{array}{ccc}
\mathbf{M}_{1} & \ldots & \mathbf{M}_{N}
\end{array}\right]\left[\begin{array}{c}
\mathbf{x}_{1} \\
\vdots \\
\mathbf{x}_{N}
\end{array}\right] \ll 0} \\
\mathbf{x}_{\theta} \gg 0, \theta=1, \ldots, N
\end{array}\right.
$$

We write the first inequality in $(\widetilde{\mathrm{S}})$ as $\mathbf{M}_{1} \mathbf{x}_{1}+\cdots+\mathbf{M}_{N} \mathbf{x}_{N}=-\mathbf{x}_{0}$, $\mathbf{x}_{0} \gg 0$, which is equivalent to the equality $\left(\mathbf{I} \mathbf{X}_{0}+\mathbf{M}_{1} \mathbf{X}_{1}+\right.$ $\left.\cdots+\mathbf{M}_{N} \mathbf{X}_{N}\right) \mathbf{e}=0$, where $\mathbf{X}_{\theta}=\operatorname{diag}\left\{\mathbf{x}_{\theta}\right\}$ has all diagonal entries positive, $\theta=0,1, \ldots, N$, and $\mathbf{e}=[1 \cdots 1]^{T} \in \mathbb{R}^{n}$. All the column representatives of $\mathscr{M}=\left\{\mathbf{I}, \mathbf{M}_{1}, \ldots, \mathbf{M}_{N}\right\}$ are $\mathbf{M}$ matrices and their determinants are nonnegative (Theorem 6.4 .6 (A1) in ([20], p. 149). Since $\operatorname{det}(\mathrm{I})>0, \mathscr{M}$ has the column- $\mathscr{W}_{0}$-property and $\operatorname{det}\left(\mathbf{I X}_{0}+\mathbf{M}_{1} \mathbf{X}_{1}+\cdots+\mathbf{M}_{N} \mathbf{X}_{N}\right) \neq 0$ for any diagonal matrix $\mathbf{X}_{\theta}$ with positive diagonal entries, $\theta=0,1, \ldots, N$, as per Theorem 7, [21]. Hence, the equality $\left(\mathbf{I X}_{0}+\mathbf{M}_{1} \mathbf{X}_{1}+\cdots+\mathbf{M}_{N} \mathbf{X}_{N}\right) \mathbf{e}=0$ is not true and $(\widetilde{\mathrm{S}})$ is not consistent. Subsequently, $(\mathrm{S})$ is consistent and inequalities $(10-\mathrm{H})$ have the solution $\mathbf{v}>0-$ as being equivalent to the algebraic system (S). Implicitly, the semipositive vector $\mathbf{v}>0$ satisfies the inequality $\left(\left(\underline{\lambda}^{H}\right)^{*} \mathbf{I}-\underline{\mathbf{A}}_{\sigma^{*}}^{H}\right)^{T} \mathbf{v} \geq 0$.

Case (1). If there exists $\boldsymbol{\sigma}^{*} \in \underline{\mathscr{C}}^{*}$ such that the matrix $\underline{\mathbf{A}}_{\sigma^{*}}^{H}$ is irreducible, then $\left(\left(\underline{\lambda}^{H}\right)^{*} \mathbf{I}-\underline{\mathbf{A}}_{\sigma^{*}}^{\bar{H}}\right)^{T}$ is a singular, irreducible M-matrix. Thus, Theorem 6.4 .16 ([20], p. 156) applied to $\left(\left(\underline{\lambda}^{H}\right)^{*} \mathbf{I}-\underline{\mathbf{A}}_{\sigma^{*}}^{H}\right)^{T} \mathbf{v} \geq 0$ shows that the only semipositive vector $\mathbf{v}>0,\|\mathbf{v}\|_{1}=1$, satisfying $(10-\mathrm{H})$ is the left eigenvector $\mathbf{w}_{L}\left(\underline{\mathbf{A}}_{\sigma^{*}}^{H}\right) \gg 0$ (that is strictly positive). Thus, there exist $\mathbf{D}=\operatorname{diag}\left\{\mathbf{w}_{L}\left(\underline{\mathbf{A}}_{\sigma^{*}}^{H}\right)\right\}>0$ diagonal and $r=\left(\underline{\lambda}^{H}\right)^{*}<0$ ensuring $\mu_{1}^{D}\left(\mathbf{A}_{\theta}\right)=\mu_{1}^{D}\left(\mathbf{A}_{\theta}^{H}\right) \leq r, \theta=1, \ldots, N$, a fact which shows that inequalities $(8-\mathrm{H})$ are satisfied. 
Case (2). If for all $\boldsymbol{\sigma}^{*} \in \underline{\mathscr{C}}^{*}$ the matrices $\underline{\mathbf{A}}_{\sigma^{*}}^{H}$ are reducible, then consider the matrix $\overline{\mathbf{E}} \in \operatorname{Int}\left(\mathbb{R}_{+}^{n \times n}\right)$ and $c>0$. Define the set of essentially positive matrices $\mathbf{A}_{\theta}^{H}(c)=\mathbf{A}_{\theta}^{H}+c \mathbf{E}$, $\theta=1, \ldots, N$, and their column representatives $\underline{\mathbf{A}}_{\sigma}^{H}(c)=$ $\underline{\mathbf{A}}_{\sigma}^{H}+c \mathbf{E}, \boldsymbol{\sigma} \in \mathscr{C}$. For any $\boldsymbol{\sigma} \in \mathscr{C}, \lambda_{\max }\left(\underline{\mathbf{A}}_{\sigma}^{H}(c)\right)$ is a continuous and increasing function with respect to $c>0$, so that for any small $\varepsilon>0$ we can define the interval $\mathscr{J}_{\varepsilon}=\left(0, c_{\varepsilon}\right]=$ $\left\{c>0 \mid \max _{\boldsymbol{\sigma} \in \mathscr{C}} \lambda_{\max }\left(\underline{\mathbf{A}}_{\sigma}^{H}(c)\right) \leq\left(\underline{\lambda}^{H}\right)^{*}+\varepsilon<0\right\}$. For any $c \in \mathscr{J}_{\varepsilon}$, introduce (similarly to $\left.(15-\mathrm{H})\right)\left(\underline{\lambda}^{H}(c)\right)^{*}=$ $\max _{\boldsymbol{\sigma} \in \mathscr{C}} \lambda_{\max }\left(\underline{\mathbf{A}}_{\sigma}^{H}(c)\right), \underline{\mathscr{C}}_{c}^{H *}=\left\{\boldsymbol{\sigma}_{c}^{*} \in \mathscr{C} \mid \lambda_{\max }\left(\underline{\mathbf{A}}_{\sigma_{c}^{*}}^{H}(c)\right)=\right.$ $\left.\left(\underline{\lambda}^{H}(c)\right)^{*}\right\}$ and notice that there exists $\sigma_{c}^{*} \in \underline{\mathscr{C}}_{c}^{H *}$ such that matrix $\underline{\mathbf{A}}_{\sigma_{c}^{*}}^{H}(c)$ is irreducible (since all $\underline{\mathbf{A}}_{\sigma}^{H}(c), \boldsymbol{\sigma} \in \mathscr{C}$ are essentially positive and, inherently, irreducible). Hence, for any $c \in \mathscr{J}_{\varepsilon}$, we apply case (1) detailed above to the inequality $(\mathbf{v}(c))^{T} \underline{\mathbf{A}}_{\sigma_{c}^{*}}^{H}(c) \leq\left(\underline{\lambda}^{H}(c)\right)^{*}(\mathbf{v}(c))^{T}$, and we show that $\mathbf{v}(c)=$ $\mathbf{w}_{L}\left(\underline{\mathbf{A}}_{\sigma_{c}^{*}}^{H}(c)\right) \gg 0, r(c)=\left(\underline{\lambda}^{H}(c)\right)^{*}<0$ solve the inequalities $\mathbf{v}^{T} \underline{\mathbf{A}}_{\theta}^{H}(c) \leq r \mathbf{v}^{T}, \theta=1, \ldots, N$. For these $\mathbf{v}(c), r(c)$, we can write $(\mathbf{v}(c))^{T} \mathbf{A}_{\theta}^{H}<(\mathbf{v}(c))^{T} \mathbf{A}_{\theta}^{H}(c) \leq r(c)(\mathbf{v}(c))^{T}, \theta=1, \ldots, N$, meaning that $\mathbf{v}(c), r(c)$ solve inequalities $(10-\mathrm{H})$. Thus, there exist $\mathbf{D}=\operatorname{diag}\left\{\mathbf{w}_{L}\left(\underline{\mathbf{A}}_{\sigma_{c}^{*}}^{H}(c)\right)\right\}>0$ diagonal and $r=\left(\underline{\lambda}^{H}(\mathrm{c})\right)^{*}<$ 0 ensuring $\mu_{1}^{\mathbf{D}}\left(\mathbf{A}_{\theta}\right)=\mu_{1}^{\mathbf{D}}\left(\mathbf{A}_{\theta}^{H}\right) \leq r, \theta=1, \ldots, N$, a fact which shows that inequalities $(8-\mathrm{H})$ are satisfied.

The analysis of both irreducible and reducible cases for $\underline{\mathbf{A}}_{\sigma^{*}}^{H}$ completes the proof.

Theorem 5. (a) There exist $\mathbf{D}>0$ diagonal and $0<r<1$ such that system (1-S) is DIES ${ }_{\infty}^{\mathrm{D}, r}$ under arbitrary switching, if and only if $0<\left(\bar{\lambda}^{S}\right)^{*}<1$.

(b) There exist $\mathbf{D}>0$ diagonal and $r<0$ such that system $(1-\mathrm{H})$ is DIES $S_{\infty}^{\mathrm{D}, r}$ under arbitrary switching, if and only if $\left(\bar{\lambda}^{H}\right)^{*}<0$.

Proof. It is similar to the proof of Theorem 4, taking into account the fact that, in accordance with Theorem 3(iii), inequalities $(8-\mathrm{S}) / /(8-\mathrm{H})$ are equivalent to inequalities $(12-\mathrm{S}) / /$ (12-H).

Remark 6. The results in Theorems 4 and 5 focus on the qualitative aspect of the DIES analysis based on the dominant eigenvalues of the representatives, in the sense of the traditional threshold values 1 for discrete-time and 0 for continuous-time dynamics.

However the proof of Theorem 4 together with Lemma 1 in [22] also allows addressing quantitative aspects, in the sense that there exist pairs $(\mathbf{v}, r)$, with $\mathbf{v} \gg 0$, satisfying inequalities (10-S), with $r \geq\left(\underline{\lambda}^{S}\right)^{*}$ as close to $\left(\underline{\lambda}^{S}\right)^{*}$ as we want, and, respectively, inequalities $(10-\mathrm{H})$, with $r \geq\left(\underline{\lambda}^{H}\right)^{*}$ as close to $\left(\underline{\lambda}^{H}\right)^{*}$ as we want. Equivalently, this means that $\left(\underline{\lambda}^{S}\right)^{*}$ and $\left(\underline{\lambda}^{H}\right)^{*}$, respectively, represent the best (fastest) contraction rate for all the invariant sets defined by inequality (8-S) and (8-H), respectively, with $p=1$. Similar reasoning supports the conclusion that $\left(\bar{\lambda}^{S}\right)^{*}$ and $\left(\bar{\lambda}^{H}\right)^{*}$, respectively, represent the best (fastest) contraction rate for all the invariant sets defined by inequality $(8-\mathrm{S})$ and $(8-\mathrm{H})$, respectively, with $p=$ $\infty$.

\subsection{Sufficient Conditions for DIES ${ }_{2}^{\mathrm{D}, r}$}

Theorem 7. (a) If $0<\left(\underline{\lambda}^{S}\right)^{*}\left(\bar{\lambda}^{S}\right)^{*}<1$, then there exist $\mathbf{D}>0$ diagonal and $0<r<\overline{1}$, such that the discrete-time system (1-S) is DIES ${ }_{2}^{\mathbf{D}, r}$ under arbitrary switching.

(b) If $\left(\underline{\lambda}^{H}\right)^{*}+\left(\bar{\lambda}^{H}\right)^{*}<0$, then there exist $\mathbf{D}>0$ diagonal and $r<0$, such that the continuous-time system $(1-\mathrm{H})$ is $\mathrm{DIES}_{2}^{\mathrm{D}, r}$ under arbitrary switching.

Proof. We give the proof only for the continuous-time case (b); the proof of discrete-time case (a) may be addressed along the same main lines, by using matrix-norm properties instead of matrix-measure properties.

If $\varepsilon>0$ is arbitrarily small, then there exist $\underline{r}$ with $\left(\underline{\lambda}^{H}\right)^{*}<\underline{r}$ and $\bar{r}$ with $\left(\bar{\lambda}^{H}\right)^{*}<\bar{r}$, such that $\underline{r}+\bar{r}=2 r=$ $\left(\underline{\lambda}^{H}\right)^{*}+\left(\overline{\bar{\lambda}}^{H}\right)^{*}+\varepsilon<0$. Since $\left(\underline{\lambda}^{H}\right)^{*}<\underline{r}$, from the proof of Theorem 4 ("if" part) we can find $\underline{\mathrm{D}}>0$ diagonal, such that $\mu_{1}\left(\underline{\mathbf{D}}^{-1} \mathbf{A}_{\theta} \underline{\mathbf{D}}\right)=\mu_{1}\left(\underline{\mathbf{D}}^{-1} \mathbf{A}_{\theta}^{H} \underline{\mathbf{D}}\right) \leq \underline{r}, \bar{\theta}=1, \ldots, N$. By a similar reasoning (relative to Theorem 5 ), we can get $\overline{\mathbf{D}}>0$ diagonal, such that $\mu_{\infty}\left(\overline{\mathbf{D}}^{-1} \mathbf{A}_{\theta} \overline{\mathbf{D}}\right)=\mu_{\infty}\left(\overline{\mathbf{D}}^{-1} \mathbf{A}_{\theta}^{H} \overline{\mathbf{D}}\right) \leq \bar{r}, \theta=1, \ldots, N$.

Now, if we use the notations $\underline{\mathbf{D}}=(\underline{\mathbf{U}})^{2}, \overline{\mathbf{D}}=(\overline{\mathbf{U}})^{2}$, we can write

$$
\begin{aligned}
& 2 r=\underline{r}+\bar{r} \geq \mu_{1}\left(\underline{\mathbf{D}}^{-1} \mathbf{A}_{\theta} \underline{\mathbf{D}}\right)+\mu_{\infty}\left(\overline{\mathbf{D}}^{-1} \mathbf{A}_{\theta} \overline{\mathbf{D}}\right) \\
& =\mu_{1}\left(\underline{\mathbf{D}}^{-1} \mathbf{A}_{\theta} \underline{\mathbf{D}}\right)+\mu_{1}\left(\overline{\mathbf{D}}\left(\mathbf{A}_{\theta}\right)^{T} \overline{\mathbf{D}}^{-1}\right) \\
& \geq \mu_{1}\left(\underline{\mathbf{D}}^{-1} \mathbf{A}_{\theta} \underline{\mathbf{D}}+\overline{\mathbf{D}}\left(\mathbf{A}_{\theta}\right)^{T} \overline{\mathbf{D}}^{-1}\right) \\
& \geq \lambda_{\max }\left(\underline{\mathbf{D}}^{-1} \mathbf{A}_{\theta} \underline{\mathbf{D}}+\overline{\mathbf{D}}\left(\mathbf{A}_{\theta}\right)^{T} \overline{\mathbf{D}}^{-1}\right) \\
& =\lambda_{\max }\left(\left(\underline{\mathbf{U}}^{-1}\right)^{2} \mathbf{A}_{\theta} \underline{\mathbf{U}}^{2}+\overline{\mathbf{U}}^{2}\left(\mathbf{A}_{\theta}\right)^{T}\left(\overline{\mathbf{U}}^{-1}\right)^{2}\right) \\
& =\lambda_{\max }\left(( \overline { \mathbf { U } } ^ { - 1 } \underline { \mathbf { U } } ) \left[\left(\underline{\mathbf{U}}^{-1}\right)^{2} \mathbf{A}_{\theta} \underline{\mathbf{U}}^{2}\right.\right. \\
& \left.\left.+\overline{\mathbf{U}}^{2}\left(\mathbf{A}_{\theta}\right)^{T}\left(\overline{\mathbf{U}}^{-1}\right)^{2}\right]\left(\overline{\mathbf{U}}^{-1} \underline{\mathbf{U}}\right)^{-1}\right) \\
& =\lambda_{\max }\left(\overline{\mathbf{U}}^{-1} \underline{\mathbf{U}}^{-1} \mathbf{A}_{\theta} \underline{\mathbf{U}} \overline{\mathbf{U}}+\underline{\mathbf{U}} \overline{\mathbf{U}}\left(\mathbf{A}_{\theta}\right)^{T} \overline{\mathbf{U}}^{-1} \underline{\mathbf{U}}^{-1}\right) \\
& =\lambda_{\max }\left((\underline{\mathbf{U}} \overline{\mathbf{U}})^{-1} \mathbf{A}_{\theta}(\underline{\mathbf{U}} \overline{\mathbf{U}})+(\underline{\mathbf{U}} \overline{\mathbf{U}})\left(\mathbf{A}_{\theta}\right)^{T}(\underline{\mathbf{U}} \overline{\mathbf{U}})^{-1}\right) \\
& =2 \mu_{2}\left((\underline{\mathbf{U}} \overline{\mathbf{U}})^{-1} \mathbf{A}_{\theta}(\underline{\mathbf{U}} \overline{\mathbf{U}})\right), \quad \theta=1, \ldots, N \text {. }
\end{aligned}
$$

Thus, we have proven the existence of $\mathbf{D}=\underline{\mathbf{U}} \overline{\mathbf{U}}>0$ diagonal and $r=(\underline{r}+\bar{r}) / 2<0$ ensuring $\mu_{2}^{\mathbf{D}}\left(\mathbf{A}_{\theta}\right) \leq r, \theta=1, \ldots, N$, a fact which shows that inequalities $(8-\mathrm{H})$ are satisfied.

Remark 8. Unlike Theorems 4 and 5, Theorem 7 expresses only a sufficient condition for DIES. It is formulated in qualitative terms by referring to the dominant eigenvalues of the representatives, as well as to the traditional threshold values 1 for discrete-time and 0 for continuous-time.

However the proofs of Theorems 4 and 7 also allow a refined formulation in quantitative terms, in the sense that, for 
$p=2$, there exist pairs $(\mathbf{D}, r)$, with $\mathbf{D}>0$, satisfying inequalities (8-S), with $r \geq \sqrt{\left(\underline{\lambda}^{S}\right)^{*}\left(\bar{\lambda}^{S}\right)^{*}}$ as close to $\sqrt{\left(\underline{\lambda}^{S}\right)^{*}\left(\bar{\lambda}^{S}\right)^{*}}$ as we want, and, respectively, inequalities (8-H), with $r \geq$ $(1 / 2)\left(\left(\underline{\lambda}^{H}\right)^{*}+\left(\bar{\lambda}^{H}\right)^{*}\right)$ as close to $(1 / 2)\left(\left(\underline{\lambda}^{H}\right)^{*}+\left(\bar{\lambda}^{H}\right)^{*}\right)$ as we want. Obviously, this formulation in quantitative terms is still limited just to sufficiency (similarly to Theorem 7). Subsequently, the corresponding value, i.e., $\sqrt{\left(\underline{\lambda}^{S}\right)^{*}\left(\bar{\lambda}^{S}\right)^{*}}$ and $(1 / 2)\left(\left(\underline{\lambda}^{H}\right)^{*}+\left(\bar{\lambda}^{H}\right)^{*}\right)$, respectively, does not necessarily represent the best (fastest) contraction rate for all the invariant sets defined by inequality $(8-\mathrm{S})$ and $(8-\mathrm{H})$, respectively, with $p=2$.

\section{Illustrative Examples and Numerical Considerations}

The theoretical results on DIES are applied to two numerical examples referring to both continuous-time (example 1) and discrete-time (example 2) switching dynamics. Examples 1 and 2 have a similar architecture, in the sense that the constituent matrices are parameterized by two parameters $a, b$. In both examples, the construction of the DIES domains corresponding to $p \in\{1,2, \infty\}$, is addressed in a dual manner, by using the older results (summarized by Section 2), in parallel with the new ones (developed by Section 3). Relevant comparisons are permitted for the applicability of Theorem 3(i) versus Theorem 4, of Theorem 3(ii) versus Theorem 7, and of Theorem 3(iii) versus Theorem 5, respectively. Moreover, these comparative discussions on the DIES domains can include information about subdomains corresponding to various values selected for the contraction rate $r<0$ (continuoustime) and $0<r<1$ (discrete-time).

Both considered examples are inspired by the literature of arbitrary switching linear systems, as detailed below. Unlike the organization of the exposition for theoretical results, in the previous sections, the current section starts with the continuous-time case-example 1 - continued by the discrete-time case-example 2. This is because example 1 presents a higher numerical complexity and requires more meticulous explanations. The computations are performed in MATLAB R2016a, running on a laptop equipped with an Intel(R) Core ${ }^{\mathrm{TM}}$ i7-4710HQ CPU @2.50 GHz x64-based processor and 16 GB RAM.

Example 1. Consider a continuous-time arbitrary switching linear system of form $(1-\mathrm{H})$ defined by the matrices

$$
\begin{aligned}
\mathscr{A} & =\left\{\mathbf{A}_{1}, \mathbf{A}_{2}, \mathbf{A}_{3}\right\} \subset \mathbb{R}^{3 \times 3}, \\
\mathbf{A}_{1} & =\left[\begin{array}{ccc}
-12 & 6 & 2 a \\
1 & -10 & 2 \\
b+1 & 3 & -10
\end{array}\right], \\
\mathbf{A}_{2} & =\left[\begin{array}{ccc}
-12 & -4 & 0 \\
-6 & -10 & -9 \\
-b & -a & -13
\end{array}\right], \\
\mathbf{A}_{3} & =\left[\begin{array}{ccc}
-9 & -2 & -8 \\
-6 & -10 & -b \\
-a & 0 & -11
\end{array}\right],
\end{aligned}
$$

where $a, b \in[1,10]$ are parameters, inspired by the numerical example presented in [23].

We are interested in finding the domain of the parameters $(a, b) \in[1,10] \times[1,10]$ that guarantees DIES for $p \in\{1,2, \infty\}$. Given a certain $p \in\{1,2, \infty\}$, the corresponding DIES domain is represented as a union of subdomains corresponding to different decreasing rates $r$, namely, $-0.25 \leq r<0$, $-0.50 \leq r<-0.25$, etc. The subdomain role is understood as a refinement of the global results with $r<0$, which can take into consideration quantitative information about the contraction of the invariant sets defined by $(5-\mathrm{H})$. For instance, if a point $\left(a^{*}, b^{*}\right)$ belongs to the $\mathrm{DIES}_{1}^{\mathrm{D}, r}$ subdomain corresponding to $-0.25 \leq r<0$, then, for the parameter values $a^{*}, b^{*}$, system $(1-\mathrm{H})$ cannot have invariant sets of form $(5-\mathrm{H})$ with $p=1$ and a decreasing rate faster than -0.25 (regardless of the considered positive definite diagonal matrix $\mathbf{D}>0$ ).

The DIES analysis is meant to permit comparisons between the employment of the already known results reproduced in Section 2 and the employment of the new results proposed by Section 3, for all the points belonging to the grid constructed for $(a, b) \in[1,10] \times[1,10]$, with a step of 0.05 . When applying the results from Section 2 for $p \in\{1,2, \infty\}$, in each point of the grid we look for the minimum value of $r$ such that there exists a positive vector $\mathbf{v} \in \mathbb{R}^{4}, \mathbf{v} \gg 0$, fulfilling $(8-\mathrm{H})$ or the particular forms derived in Theorem 3, namely, $(10-\mathrm{H}),(11-\mathrm{H})$, and $(12-\mathrm{H})$, respectively. This problem can be solved numerically by using the fmincon solver from the Optimization Toolbox for MATLAB or the ga solver from the Global Optimization Toolbox for MATLAB, that performs the minimization of a function subject to nonlinear constraints. For the particular case when $p=2$, the constraints (11-H) are in the form of BMIs (bilinear matrix inequalities) and may be solved, for example, using the PENBMI solver commercially available from TOMLAB Optimization [24]. A second strategy that can be employed uses a bisection strategy for $r \in\left[\max \left\{\lambda_{\max }\left(\mathbf{A}_{1}^{H}\right), \lambda_{\max }\left(\mathbf{A}_{2}^{H}\right), \lambda_{\max }\left(\mathbf{A}_{3}^{H}\right)\right\}, 10\right]$ that yields (for every feasible $r$ ) a linear programming problem for $p \in$ $\{1, \infty\}$ that can be approached using the linprog solver from the Optimization Toolbox for MATLAB or an LMI (linear matrix inequality) problem for $p=2$ that can be solved using CVX, a MATLAB package for specifying and solving convex programs $[25,26]$. In the current paper we have adopted the first strategy depicted previously. The numerical implementation of the results from Section 3 requires standard linear algebra facilities for the representative construction and eigenvalue computation using MATLAB built-in functions.

Let us begin the DIES analysis for $p=1$. The DIES ${ }_{1}^{\mathrm{D}, r}$ parametric domain in Figure 1(a) is obtained applying Theorems 2 and 3(i). The DIES ${ }_{1}^{\mathrm{D}, r}$ parametric domain in Figure 1(b) was obtained applying Theorem 4(b) and Remark 6. In each point of the grid, we compute the dominant eigenvalue $\left(\underline{\lambda}^{H}\right)^{*}$ for all column representatives of the matrix family

$$
\begin{aligned}
& \mathscr{A}^{H}=\left\{\mathbf{A}_{1}^{H}, \mathbf{A}_{2}^{H}, \mathbf{A}_{3}^{H}\right\} \subset \mathbb{R}^{3 \times 3}, \\
& \mathbf{A}_{1}^{H}=\left[\begin{array}{ccc}
-12 & 6 & 2 a \\
1 & -10 & 2 \\
b+1 & 3 & -10
\end{array}\right],
\end{aligned}
$$




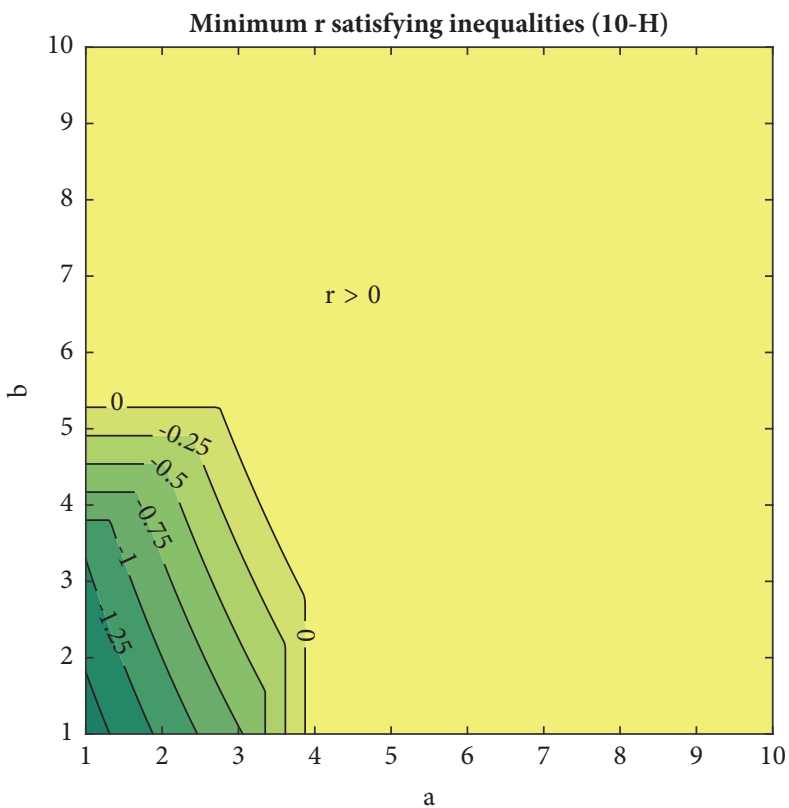

(a)

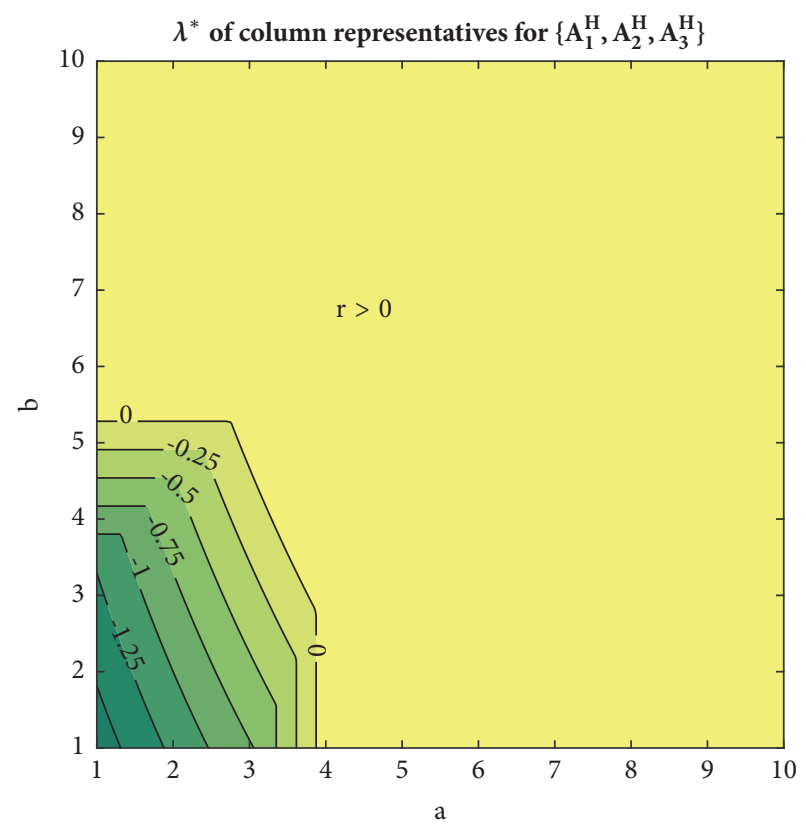

(b)

FIGURE 1: Comparative plots of DIES $S_{1}^{\mathrm{D}, r}$ parametric domain for example 1 provided by (a) the MATLAB implementation of the old results summarized in Section 2 (Theorems 2 and 3(i)); (b) the MATLAB implementation of the new results developed in Section 3 (Theorem 4(b) and Remark 6).

$$
\begin{aligned}
& \mathbf{A}_{2}^{H}=\left[\begin{array}{ccc}
-12 & 4 & 0 \\
6 & -10 & 9 \\
b & a & -13
\end{array}\right], \\
& \mathbf{A}_{3}^{H}=\left[\begin{array}{ccc}
-9 & 2 & 8 \\
6 & -10 & b \\
a & 0 & -11
\end{array}\right] .
\end{aligned}
$$

The plot presented in Figure 1(b) offers the same graphic information as Figure 1(a)-a fact expected from the theoretical point of view, in accordance with the proof of Theorem 4 and the comments included in Remark 6. Nevertheless, it is extremely important to notice that the computation effort corresponding to the old approach (summarized in Section 2) is significantly greater than the effort corresponding to the new one (developed in Section 3). For the considered example (studied on the machine with the characteristics presented above), the requested computation time is 8,826 seconds for the first (old) procedure and 10.85 seconds for the second (new) one. This major difference is caused by the great number of points in the grid, but even at the level of a single point the faster operation of the new procedure is directly visible. This fact may be regarded as a general advantage of the new approach, not strictly related to the considered example. However, generally speaking, the cardinality of the columnrepresentative set associated with $\mathscr{A}^{H}$ must be reasonable, because the existence of a high number of representatives may severely increase the computation time for $\left(\underline{\lambda}^{H}\right)^{*}$.
Here we may also point out an interesting connection with the numerical example from paper [23] that corresponds to the arbitrary switching positive system defined by the essentially nonnegative matrices (21), with the particular values $a \in\{3,5\}$ and $b=4$. The cited example studies the stability by means of copositive linear Lyapunov functions, an investigation which is equivalent to DIES ${ }_{1}$ analysis of the switching system defined by the matrices (20). Our results are in agreement with the findings in [23]. For $a=3$ and $b=4$, the switching system defined by matrices (20) is DIES $_{1}$, meaning the existence of a common copositive linear Lyapunov function for the switching positive system defined by matrices (21). For $a=5$ and $b=4$ the switching system defined by matrices (20) is not DIES ${ }_{1}$, meaning that no common copositive linear Lyapunov function can be found for the switching positive system defined by matrices (21).

For $p=\infty$ the DIES analysis is addressed mutatis mutandis along the same lines as the previously discussed case of $p=1$. The old procedure relies on the use of Theorems 2 and 3(iii) in each point of the grid and provides the DIES $S_{\infty}^{\mathrm{D}, r}$ parametric domain in Figure 2(a). The new procedure applies Theorem 5(b) and Remark 6 in each point of the grid and computes the dominant eigenvalue $\left(\bar{\lambda}^{H}\right)^{*}$ for all row representatives of the matrix family $\mathscr{A}^{H}=\left\{\mathbf{A}_{1}^{H}, \mathbf{A}_{2}^{H}, \mathbf{A}_{3}^{H}\right\}$; the resulting DIES ${ }_{\infty}^{\mathrm{D}, r}$ parametric domain is depicted in Figure 2(b). The substantial difference between the computation times requested by the two methods (commented above for $p=1$ ) is also evident here, 9,026 seconds being necessary for the first (old) procedure and 10.89 seconds for the second (new) one. 


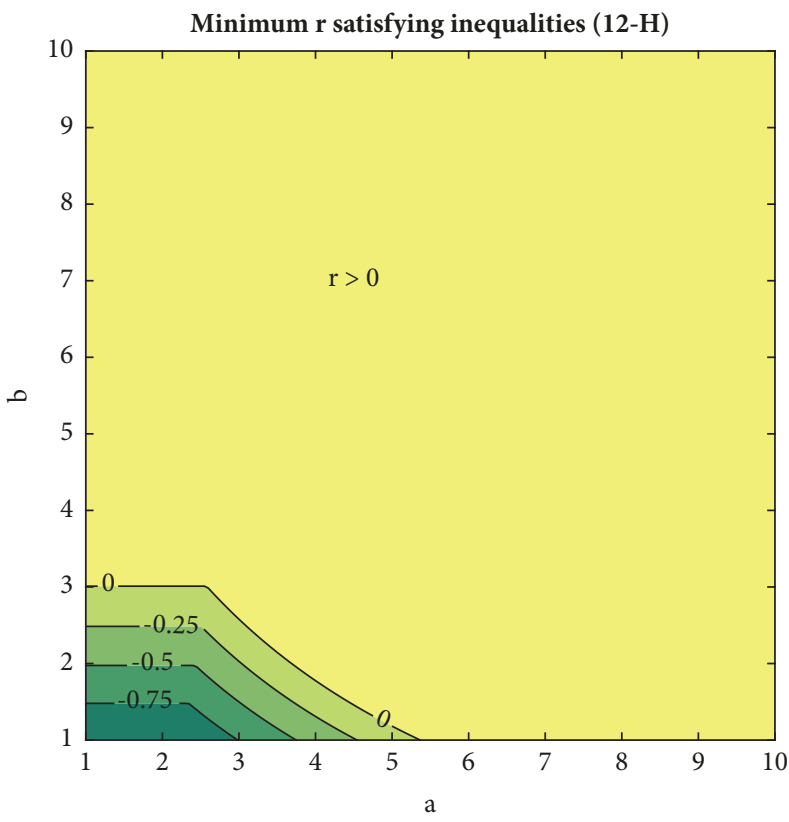

(a)

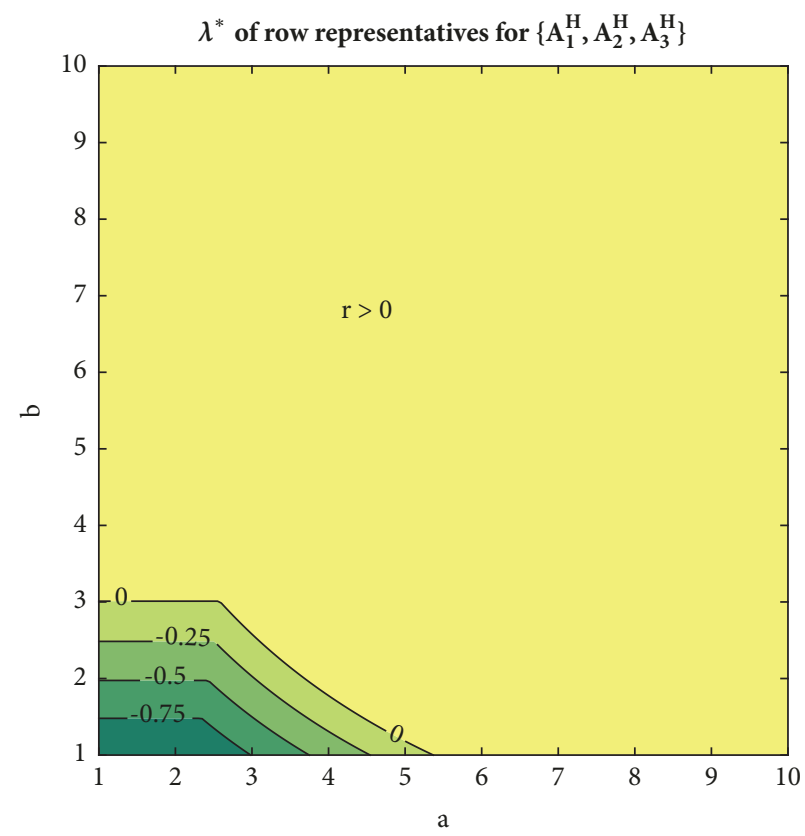

(b)

FIgURE 2: Comparative plots of DIES $S_{\infty}^{\mathbf{D}, r}$ parametric domain for example 1 provided by (a) the MATLAB implementation of the old results summarized in Section 2 (Theorems 2 and 3(iii)); (b) the MATLAB implementation of the new results developed in Section 3 (Theorem 5(b) and Remark 6).

The DIES analysis for $p=2$ reveals noticeable variations in the structure of the DIES $S_{2}^{\mathrm{D}, r}$ parametric domains provided by the old results summarized in Section 2 (i.e., Theorems 2 and 3(ii)) and by the new results developed in Section 3 (i.e., Theorem 7(b) and Remark 8). Theorems 2 and 3(ii) are applied in each point of the grid and provide the domain presented in Figure 3(a). Theorem 7(b) and Remark 8 are applied in each point of the grid for computing $\left(\underline{\lambda}^{H}\right)^{*}$ associated with all column representatives and $\left(\bar{\lambda}^{H}\right)^{*}$ associated with all row representatives, as well as the value $\left(\underline{\lambda}^{H}\right)^{*}+\left(\bar{\lambda}^{H}\right)^{*}$. The resulting domain is presented in Figure 3(b) and one can simply notice that the $\mathrm{DIES}_{2}^{\mathrm{D}, r}$ region in this figure is included into (but not identical to) the $\operatorname{DIES}_{2}^{\mathrm{D}, r}$ region in Figure 3(a). This is because Theorem 7(b) expresses just a sufficient condition, unlike the necessary and sufficient condition formulated by Theorems 2 and 3(ii), a fact already mentioned in Remark 8. On the other hand, the advantage of a much shorter computation time requested by the new procedure, compared to the old one, still exists (as in the previously studied cases of $p \in\{1, \infty\}$ ). The concrete duration for this example is 20,652 seconds for the first approach and 21.75 seconds for the second one. Under such circumstances, the use of the new procedure may be preferable for some types of $\mathrm{DIES}_{2}^{\mathrm{D}, r}$ problems, compared to the old procedure, despite the inconvenience of the new one that ensures only sufficiency.

This example, via Figure 3, shows that relatively large differences can occur for $\operatorname{DIES}_{2}^{\mathrm{D}, r}$ when using the sufficient condition in Theorem 7, unlike the necessary and sufficient condition in Theorem 3(ii). Generally speaking, these differences cannot be anticipated from the simple examination of the switching system model. For instance our example 2 illustrates a visibly smaller difference between the applications of the two theorems.

Example 2. Consider a discrete-time arbitrary switching linear system of form (1-S) defined by the matrices

$$
\begin{aligned}
\mathscr{A} & =\left\{\mathbf{A}_{1}, \mathbf{A}_{2}\right\}, \\
\mathbf{A}_{1} & =\left[\begin{array}{cc}
a & 0.8 \\
0.3 & 0.3
\end{array}\right], \\
\mathbf{A}_{2} & =\left[\begin{array}{cc}
b & 0.2 \\
0.6 & 0.2
\end{array}\right],
\end{aligned}
$$

where $a, b \in[0,1]$ are parameters, inspired by the numerical example presented in [27]. Note that matrices $\mathbf{A}_{1}, \mathbf{A}_{2}$ (22) are nonnegative, and therefore $\mathbf{A}_{\theta}^{S}=\mathbf{A}_{\theta}, \theta=1,2$; i.e., $\mathscr{A}^{S}=\mathscr{A}$. The DIES analysis is organized mutatis mutandis as in example 1 , by taking a grid constructed for $(a, b) \in$ $[0,1] \times[0,1]$, with a step of 0.02 .

Figures 4 and 5 present the DIES ${ }_{1}^{\mathrm{D}, r}$ and the DIES $S_{\infty}^{\mathrm{D}, r}$ parametric domain, respectively, obtained by applying Theorems 4(a) and 4(a), whose numerical implementation required 0.115 seconds and 0.105 seconds, respectively. As mentioned in the comment on example 1, the application of Theorem 2 together with Theorem 3(i) or 2(iii) would provide the same domains but at the expense of much longer computation times. 


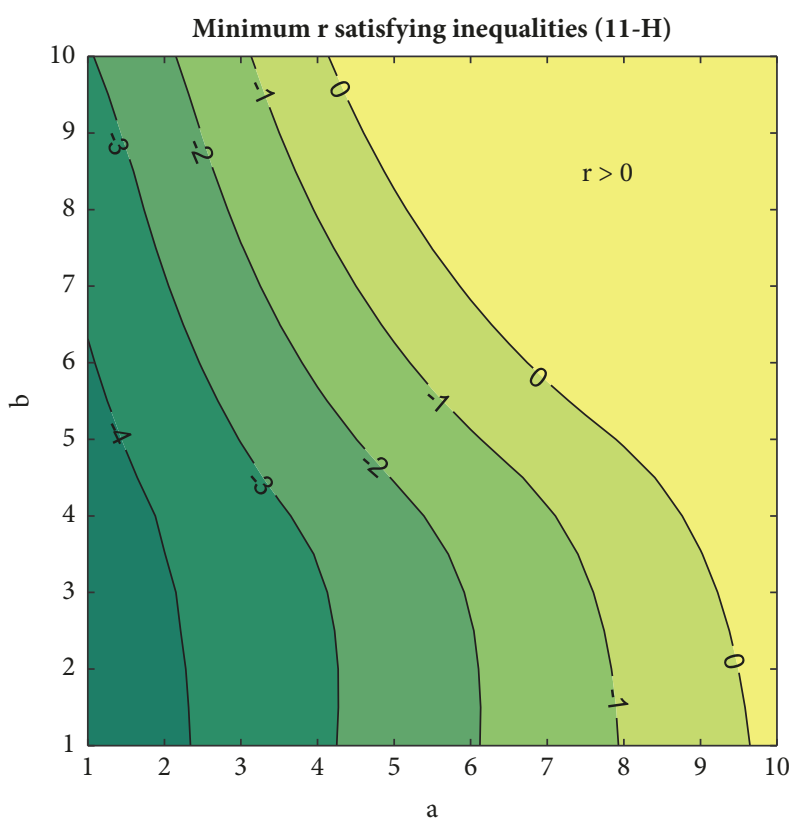

(a)

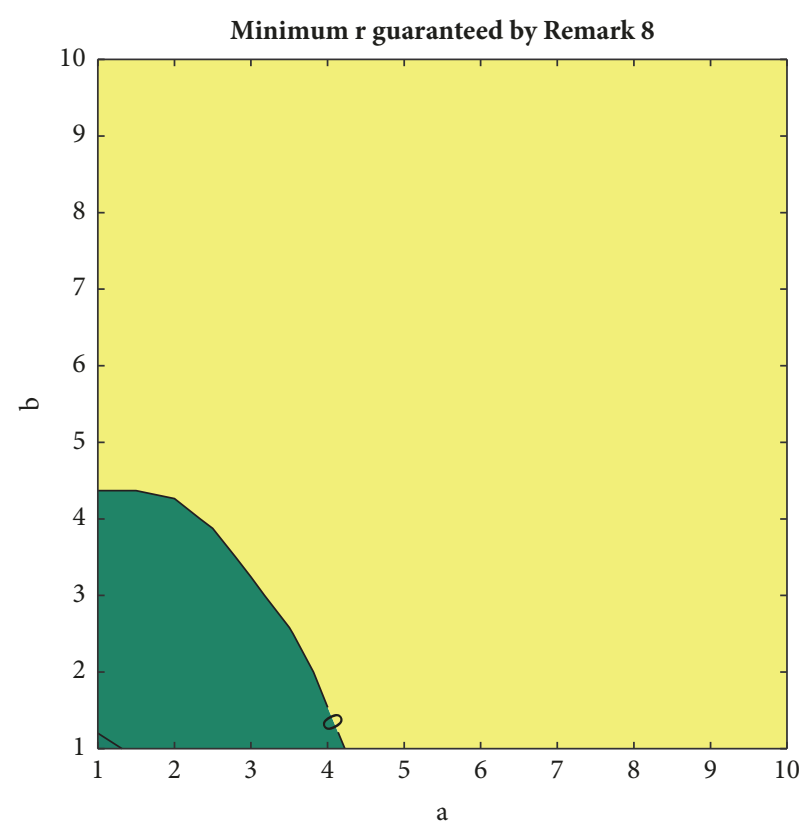

(b)

FIGURE 3: Comparative plots of $\mathrm{DIES}_{2}^{\mathrm{D}, r}$ parametric domain for example 1 provided by (a) the MATLAB implementation of the old results summarized in Section 2 (Theorems 2 and 3(ii)); (b) the MATLAB implementation of the new results developed in Section 3 (Theorem 7(b) and Remark 8).

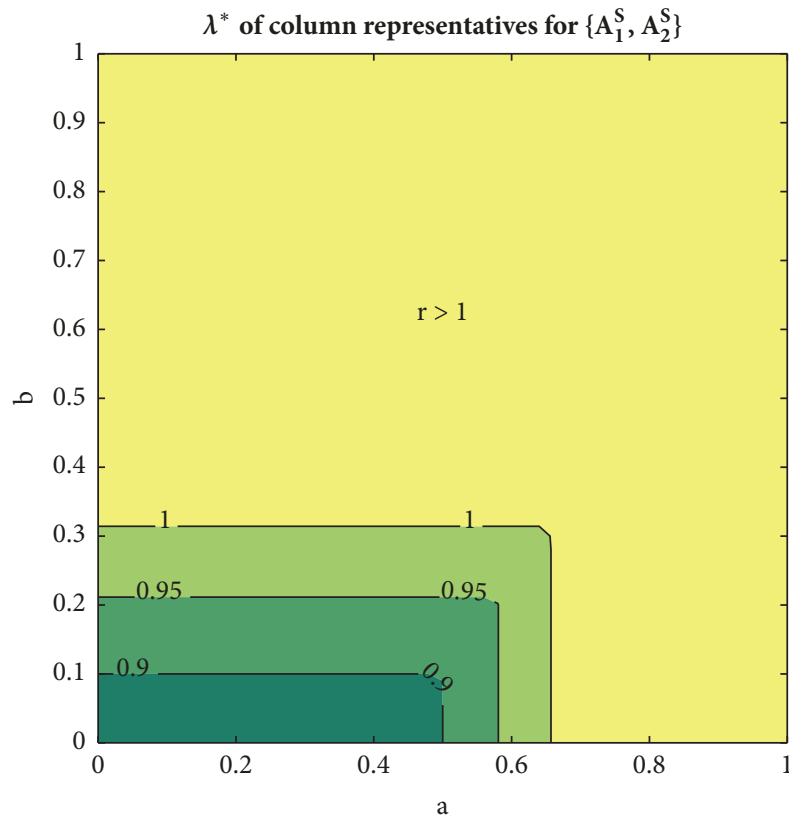

Figure 4: Plot of DIES ${ }_{1}^{\mathbf{D}, r}$ parametric domain for example 2 provided by the MATLAB implementation of Theorem 4(a) and Remark 6.

The application of Theorem 2 together with Theorem 3(ii) yields the DIES ${ }_{2}^{\mathrm{D}, r}$ parametric domain depicted in Figure 6(a) and requires a computation time of 765.9 seconds. On the other hand, the application of Theorem 7 requires only 0.23 seconds and yields the DIES ${ }_{2}^{\mathrm{D}, r}$ parametric domain depicted

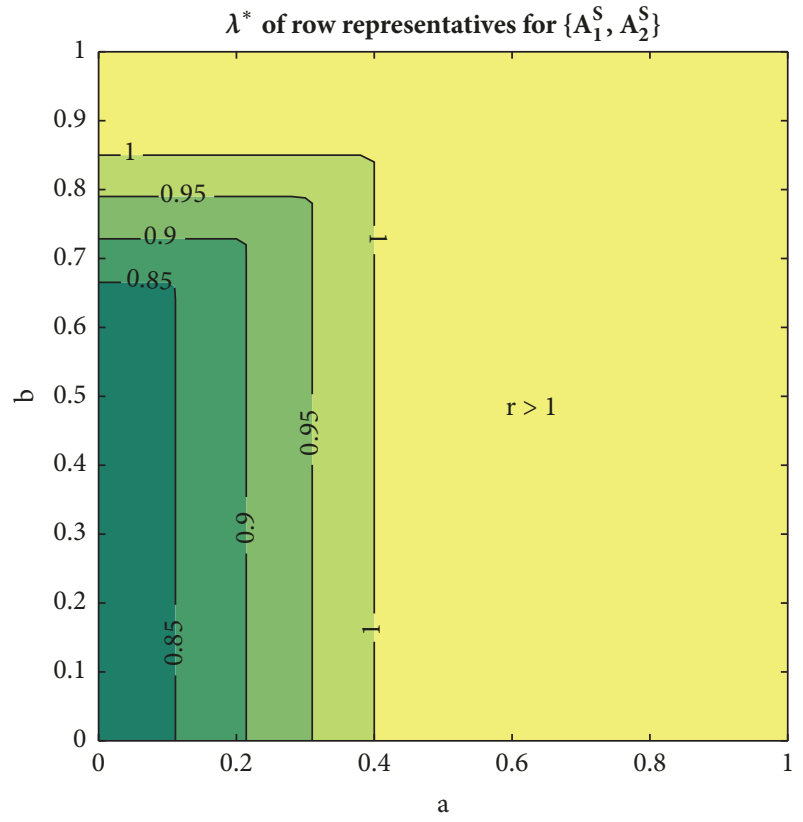

FIGURE 5: Plot of DIES ${ }_{\infty}^{\mathrm{D}, r}$ parametric domain for example 2 provided bythe MATLAB implementation of Theorem 5(a) and Remark 6 .

in Figure 6(b). A direct visual inspection of Figures 3 and 6 shows that the differences between the $\mathrm{DIES}_{2}^{\mathrm{D}, r}$ parametric domains provided by the two theorems (Theorem 7 versus Theorem 3(ii)) is significantly smaller in example 2 than in example 1 . This comparison consolidates our point of view 


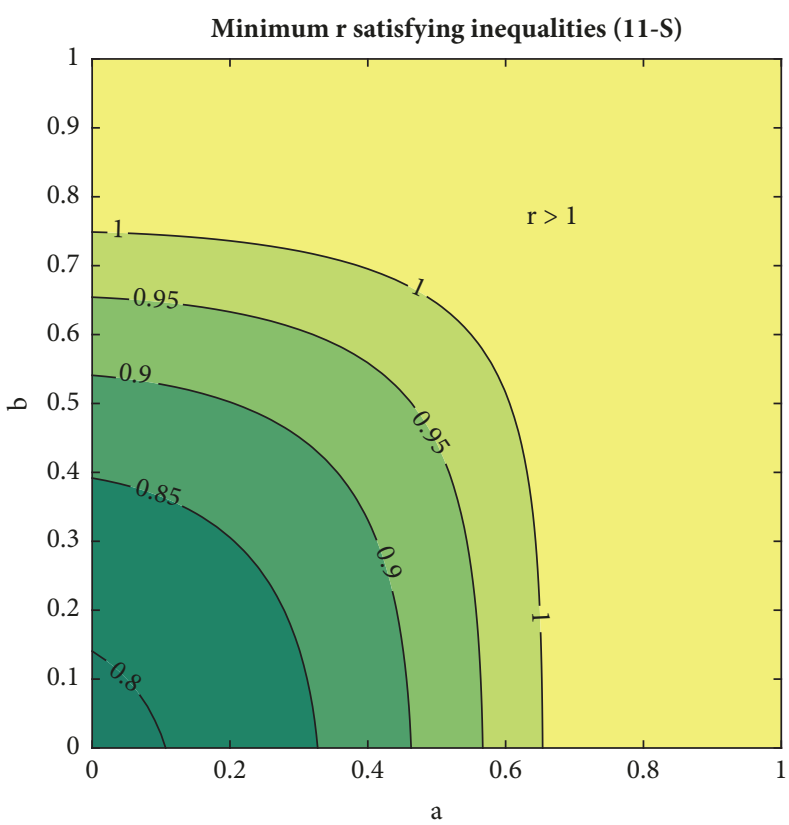

(a)

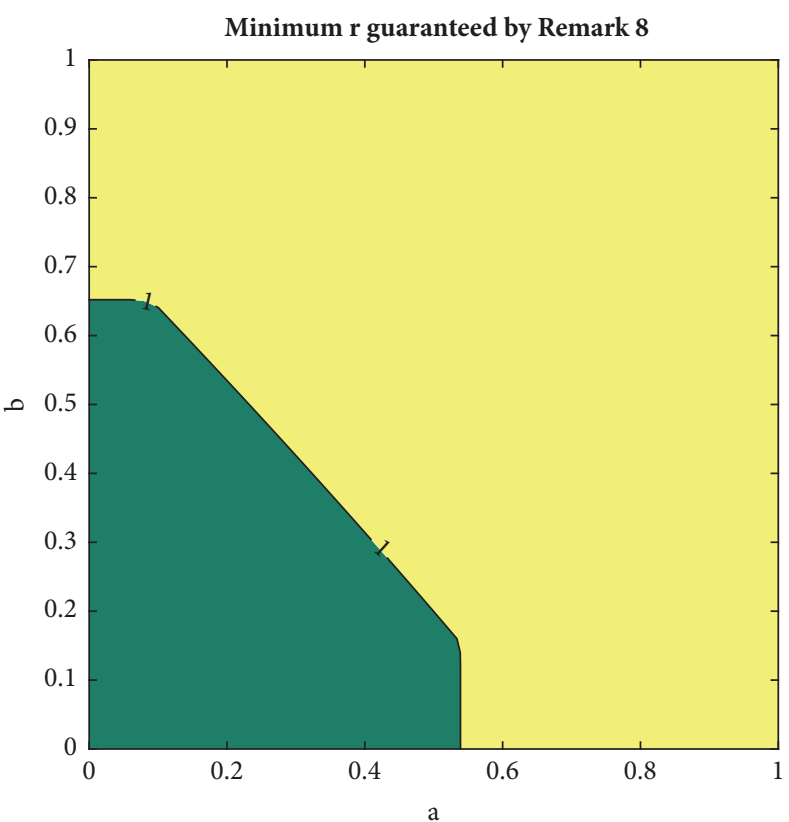

(b)

FIGURE 6: Comparative plots of DIES ${ }_{2}^{\mathrm{D}, r}$ parametric domain for example 2 provided by (a) the MATLAB implementation of the old results summarized in Section 2 (Theorems 2 and 3(ii)); (b) the MATLAB implementation of the new results developed in Section 3 (Theorem 7(a) and Remark 8).

on the practical usefulness of Theorem 7, offering a DIES ${ }_{2}^{\text {D, } r}$ sufficient condition, whose computational advantages cannot be ignored.

\section{Conclusions}

The current paper presents a novel approach to the characterization of DIES ${ }_{p}^{\mathrm{D}, r}$ with $p \in\{1,2, \infty\}$, for arbitrary switching linear systems modelled by $(1-\mathrm{S}) / /(1-\mathrm{H})$ and $(2)$, in both discrete- and continuous-time, based on the usage of row and column representatives built for the set of nonnegative matrices $\mathscr{A}^{S}$ (discrete-time case) and $\mathscr{A}^{H}$ (continuous-time case), respectively. This approach explores a theoretical potential that has not been exploited by previous works, whose main results are stated in Theorems 2 and 3. For $p \in\{1, \infty\}$ Theorems 4 and 5 provide necessary and sufficient conditions for DIES ${ }_{p}^{\mathrm{D}, r}$, whereas for $p=2$ the condition presented in Theorem 7 is only sufficient for DIES $_{2}^{\mathbf{D}, r}$. The applicability of the results is illustrated for concrete numerical examples which also compare similarities and differences between the current approach and the older results depicted in Theorems 2 and 3. At the same time, these comparisons highlight a number of advantages referring to the computational effort required by the numerical implementation based on the new results paralleled to previous ones. The use of representatives theory in $\mathrm{DIES}_{p}^{\mathrm{D}, r}$ analysis for $p \in\{1,2, \infty\}$ is expected to be also applicable to linear dynamics with polytopic uncertainties. Our further research will focus on this class of systems, aimed at the possible development of results relying on the same principles as proposed by the current paper for switching linear systems.

\section{Appendix}

\section{A. Notations and Nomenclature}

A.1. Matrix Norms, Measures, and Inequalities [28]. For a vector $\mathbf{x}=\left[\begin{array}{llll}x_{1} & x_{2} & \cdots & x_{n}\end{array}\right]^{T} \in \mathbb{R}^{n},\|\mathbf{x}\|_{p}$ is the Hölder vector $p$-norm defined by $\|\mathbf{x}\|_{p}=\left(\sum_{i=1}^{n}\left|x_{i}\right|^{p}\right)^{1 / p}$ for $1 \leq p<$ $\infty$ and $\|\mathbf{x}\|_{\infty}=\max _{i \in\{1, \ldots, n\}}\left|x_{i}\right|$ for $p=\infty$. For a square matrix $\mathbf{M}=\left[m_{i j}\right] \in \mathbb{R}^{n \times n}$, the matrix norm induced by the vector norm \|\|$_{p}$ is $\|\mathbf{M}\|_{p}=\sup _{\mathbf{y} \in \mathbb{R}^{n}, \mathbf{y} \neq 0}\|\mathbf{M y}\|_{p} /\|\mathbf{y}\|_{p}=$ $\max _{\mathbf{y} \in \mathbb{R}^{n},\|\mathbf{y}\|_{p}=1}\|\mathbf{M y}\|_{p}$, and $\mu_{p}(\mathbf{M})=\lim _{\theta \downarrow 0}\left(\|\mathbf{I}+\theta \mathbf{M}\|_{p}-1\right) / \theta$ is the corresponding matrix measure (logarithmic norm).

For a square matrix $\mathbf{M} \in \mathbb{R}^{n \times n}, \lambda_{i}(M) \in \mathbb{C}, i=1, \ldots, n$, denote its eigenvalues; if $\mathbf{M}$ is symmetric, then $\lambda_{i}(\mathbf{M}) \in \mathbb{R}$, $i=1, \ldots, n$.

Given $\mathbf{D}=\operatorname{diag}\left\{d_{1}, \ldots, d_{n}\right\}$, a positive definite diagonal matrix (i.e., $d_{i}>0, i=1, \ldots n$ ), we define the vector norm $\|\mathbf{x}\|_{p}^{\mathbf{D}}=\left\|\mathbf{D}^{-1} \mathbf{x}\right\|_{p}$, the induced matrix norm $\|\mathbf{M}\|_{p}^{\mathbf{D}}=$ $\left\|\mathbf{D}^{-1} \mathbf{M D}\right\|_{p}$, and the corresponding matrix measure $\mu_{p}^{D}(\mathbf{M})=$ $\lim _{\theta \downarrow 0}(1 / \theta)\left(\|\mathbf{I}+\theta \mathbf{M}\|_{p}^{\mathbf{D}}-1\right)$. The particular cases when $p \in$ $\{1,2, \infty\}$ are

$$
\begin{aligned}
\|\mathbf{M}\|_{1}^{\mathbf{D}} & =\max _{j \in\{1, \ldots, n\}} \sum_{i=1}^{n} \frac{d_{j}}{d_{i}}\left|m_{i j}\right|, \\
\mu_{1}^{\mathbf{D}}(\mathbf{M}) & =\max _{j \in\{1, \ldots, n\}}\left\{m_{j j}+\sum_{i=1, i \neq j}^{n} \frac{d_{j}}{d_{i}}\left|m_{i j}\right|\right\},
\end{aligned}
$$




$$
\begin{aligned}
\|\mathbf{M}\|_{2}^{\mathbf{D}} & =\sqrt{\max _{i \in\{1, \ldots, n\}}\left\{\lambda_{i}\left(\mathbf{D M}^{T}\left(\mathbf{D}^{-1}\right)^{2} \mathbf{M} \mathbf{D}\right)\right\}}, \\
\mu_{2}^{\mathbf{D}}(\mathbf{M}) & =\frac{1}{2} \max _{i \in\{1, \ldots, n\}}\left\{\lambda_{i}\left(\mathbf{D}^{-1} \mathbf{M} \mathbf{D}+\mathbf{D M}^{T} \mathbf{D}^{-1}\right)\right\} \\
\|\mathbf{M}\|_{\infty}^{\mathbf{D}} & =\max _{i \in\{1, \ldots, n\}} \sum_{j=1}^{n} \frac{d_{j}}{d_{i}}\left|m_{i j}\right|, \\
\mu_{\infty}^{\mathbf{D}}(\mathbf{M}) & =\max _{i \in\{1, \ldots, n\}}\left\{m_{i i}+\sum_{j=1, j \neq i}^{n} \frac{d_{j}}{d_{i}}\left|m_{i j}\right|\right\} .
\end{aligned}
$$

For both vectors and matrices, $\mathbf{X}^{T}$ denotes transposition, and the inequalities $\mathbf{X}_{1} \leq \mathbf{X}_{2}, \mathbf{X}_{1}<\mathbf{X}_{2}$ operate elementwise.

If matrix $\mathbf{M} \in \mathbb{R}^{n \times n}$ is symmetric, then $\mathbf{M} \succ 0(\mathbf{M} \preceq 0)$ means $\mathbf{M}$ is positive definite (negative semidefinite).

A.2. (Essentially) Nonnegative and Positive Matrices [20]. A rectangular matrix $\mathbf{X}=\left[x_{i j}\right] \in \mathbb{R}^{n \times m}$ is called (i) nonnegative (notation $\mathbf{X} \geq 0$ ) if $\mathbf{X} \in \mathbb{R}_{+}^{n \times m} \Longleftrightarrow x_{i j} \geq 0, i=1, \ldots, n$, $j=1, \ldots, m$; (ii) semipositive (notation $\mathbf{X}>0$ ) if $\mathbf{X} \geq 0$ and $\mathbf{X} \neq 0$; (iii) positive (notation $\mathbf{X} \gg 0$ ) if $\mathbf{X} \in \operatorname{Int}\left(\mathbb{R}_{+}^{n \times m}\right) \Longleftrightarrow$ $x_{i j}>0, i=1, \ldots, n, j=1, \ldots, m$.

A square matrix $\mathbf{X}=\left[x_{i j}\right] \in \mathbb{R}^{n \times n}$ is called essentially nonnegative (positive) if $x_{i j} \geq 0,\left(x_{i j}>0\right), i, j=1, \ldots, n$, $i \neq j$.

A.3. Eigenstructure of (Essentially) Nonnegative Matrices [20]. Let $\mathbf{M} \in \mathbb{R}^{n \times n}$ be (essentially) nonnegative and let $\lambda_{i}(\mathbf{M})$, $i=1, \ldots, n$, denote its eigenvalues. (i) $\mathbf{M}$ nonnegative has a real eigenvalue $\lambda_{\max }(\mathbf{M})$ such that $\left|\lambda_{i}(\mathbf{M})\right| \leq \lambda_{\max }(\mathbf{M})$, $i=1, \ldots, n$. (ii) $\mathbf{M}$ essentially nonnegative has a real eigenvalue $\lambda_{\text {max }}(\mathbf{M})$, such that $\operatorname{Re}\left\{\lambda_{i}(\mathbf{M})\right\} \leq \lambda_{\text {max }}(\mathbf{M}), i=1, \ldots, n$. (iii) $\mathbf{M}$ (essentially) nonnegative has a nonnegative right eigenvector $\mathbf{w}_{R}(\mathbf{M})>0$, satisfying $\left\|\mathbf{w}_{R}(\mathbf{M})\right\|_{1}=1$, and a nonnegative left eigenvector $\mathbf{w}_{L}(\mathbf{M})>0$, satisfying $\left\|\mathbf{w}_{L}(\mathbf{M})\right\|_{1}=1$, that correspond to the eigenvalue $\lambda_{\text {max }}(\mathbf{M})$. (iv) $\mathbf{M}$ (essentially) nonnegative and irreducible (i.e., its associated graph is strongly connected) has $\lambda_{\text {max }}(\mathbf{M})$ simple eigenvalue and $\mathbf{w}_{R}(\mathbf{M}) \gg 0, \mathbf{w}_{L}(\mathbf{M}) \gg 0$ (Perron-Frobenius eigenstructure).

$\mathbf{M} \in \mathbb{R}^{n \times n}$ is called an M-matrix if it has the form $\mathbf{M}=$ $s \mathbf{I}-\mathbf{X}, \mathbf{X} \geq 0, s \geq \lambda_{\max }(\mathbf{X})$.

\section{Data Availability}

All data regarding the numerical examples included in the article are available upon request.

\section{Disclosure}

The research was performed as part of the authors' employment at the "Gheorghe Asachi" Technical University of Iasi, Romania.

\section{Conflicts of Interest}

The authors declare that there are no conflicts of interest regarding the publication of this paper.

\section{References}

[1] D. Liberzon, Switching in Systems and Control, Birkhauser, Boston, 2003.

[2] H. Lin and P. J. Antsaklis, "Stability and stabilizability of switched linear systems: a survey of recent results," IEEE Transactions on Automatic Control, vol. 54, no. 2, pp. 308-322, 2009.

[3] M. S. Mahmoud, Switched Time-Delay Systems, Springer, Boston, Mass, USA, 2010.

[4] Z. Sun and S. S. Ge, Stability Theory of Switched Dynamical Systems, Communications and Control Engineering Series, Springer-Verlag, London, 2011.

[5] F. Blanchini, D. Casagrande, and S. Miani, "Modal and transition dwell time computation in switching systems: a settheoretic approach," Automatica, vol. 46, no. 9, pp. 1477-1482, 2010.

[6] G. Chesi, P. Colaneri, J. C. Geromel, R. Middleton, and R. Shorten, "A nonconservative LMI condition for stability of switched systems with guaranteed dwell time," Institute of Electrical and Electronics Engineers Transactions on Automatic Control, vol. 57, no. 5, pp. 1297-1302, 2012.

[7] A. T. Koru, A. Delibaşı, and H. Özbay, "On dwell time minimization for switched delay systems: Free-weighting matrices method," in Proceedings of the IEEE 53rd Annual Conference on Decision and Control (CDC), pp. 1978-1982, Los Angeles, CA, USA, December 2014.

[8] S. Y. Çalişkan, H. Őzbay, and S.-I. Niculescu, "Dwell-time computation for stability of switched systems with time delays," IET Control Theory \& Applications, vol. 7, no. 10, pp. 1422-1428, 2013.

[9] Z. Li, H. Gao, R. Agarwal, and O. Kaynak, "H1 control of switched delayed systems with average dwell time," International Journal of Control, vol. 86, no. 12, pp. 2146-2158, 2013.

[10] J. Zhang, Z. Han, F. Zhu, and J. Huang, "Stability and stabilization of positive switched systems with mode-dependent average dwell time," Nonlinear Analysis: Hybrid Systems, vol. 9, pp. 4255, 2013.

[11] J. Zhang, J. Huang, and X. Zhao, "Further results on stability and stabilisation of switched positive systems," IET Control Theory \& Applications, vol. 9, no. 14, pp. 2132-2139, 2015.

[12] F. Blanchini, P. Colaneri, and M. E. Valcher, "Switched Positive Linear Systems," Foundations and Trends in Systems and Control, vol. 2, no. 2, pp. 101-273, 2015.

[13] O. Pastravanu and M. Voicu, "Generalized matrix diagonal stability and linear dynamical systems," Linear Algebra and its Applications, vol. 419, no. 2-3, pp. 299-310, 2006.

[14] O. Pastravanu and M.-H. Matcovschi, "Diagonal stability of interval matrices and applications," Linear Algebra and its Applications, vol. 433, no. 8-10, pp. 1646-1658, 2010.

[15] M.-H. Matcovschi and O. Pastravanu, "Exponentially contractive invariant sets for discrete-time switching linear systems," in Proceedings of the 20th Mediterranean Conference on Control and Automation, MED '12, pp. 813-818, Barcelona, Spain, July 2012.

[16] M.-H. Matcovschi and O. Pastravanu, "Diagonally invariant exponential stability and stabilizability of switching linear systems," Mathematics and Computers in Simulation, vol. 82, no. 8, pp. 1407-1418, 2012. 
[17] H. Zou, H. Su, and J. Chu, "Matrix Measure Stability Criteria for a class of switched linear systems," in Intelligent Control and Automation, International Conference on Intelligent Computing ICIC, LNCIS, D.-S. Huang, K. Li, and G. W. Irwin, Eds., vol. 344, pp. 407-416, Berlin Heidelberg, 2006.

[18] Y. Ebihara, D. Peaucelle, and D. Arzelier, S-Variable Approach to LMI-based Robust Control, Springer, 2015.

[19] J. G. VanAntwerp and R. D. Braatz, "Tutorial on linear and bilinear matrix inequalities," Journal of Process Control, vol. 10, no. 4, pp. 363-385, 2000.

[20] A. Berman and R. J. Plemmons, Plemmons, Nonnegative Matrices in the Mathematical Sciences, vol. 9, SIAM Academic Press, San Diego, Calif, USA, 1994.

[21] R. Sznajder and M. S. Gowda, "Generalizations of P0- and Pproperties; extended vertical and horizontal linear complementarity problems," Linear Algebra and its Applications, vol. 223224, no. C, pp. 695-715, 1995.

[22] O. C. Pastravanu and M.-H. Matcovschi, "Max-type copositive Lyapunov functions for switching positive linear systems," Automatica, vol. 50, no. 12, pp. 3323-3327, 2014.

[23] F. Knorn, O. Mason, and R. Shorten, "On linear co-positive Lyapunov functions for sets of linear positive systems," Automatica, vol. 45, no. 8, pp. 1943-1947, 2009.

[24] TOMLAB/PENBMI Homepage, https://tomopt.com/tomlab/ products/penbmi/, 2017.

[25] M. Grant and S. Boyd, "Graph implementations for nonsmooth convex programs," in Recent Advances in Learning and Control (a tribute to M. Vidyasagar), V. Blondel, S. Boyd, and H. Kimura, Eds., vol. 371, pp. 95-110, Springer, 2008.

[26] M. Grant and S. Boyd, CVX: Matlab software for disciplined convex programming, Version 2.1, Build 1123, December 2017, http://cvxr.com/cvx.

[27] X. Liu, "Stability analysis of switched positive systems: A switched linear copositive Lyapunov function method," IEEE Transactions on Circuits and Systems II: Express Briefs, vol. 56, no. 5, pp. 414-418, 2009.

[28] D. S. Bernstein, Matrix Mathematics: Theory, Facts, and Formulas, Princeton University Press, Princeton, NJ, USA, 2009. 


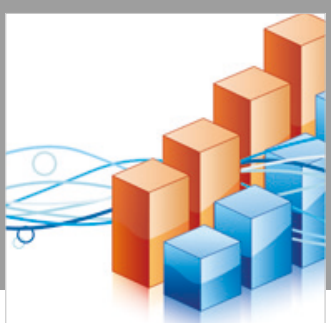

Advances in

Operations Research

\section{-n-m}
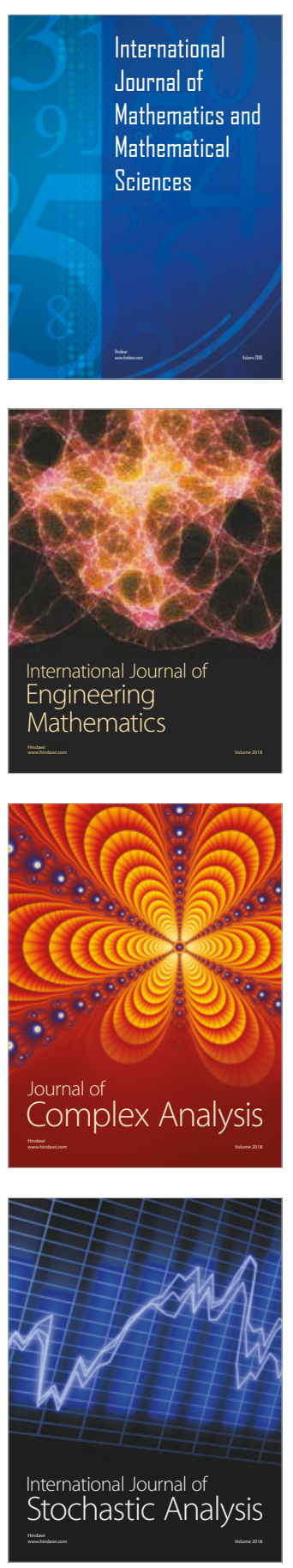
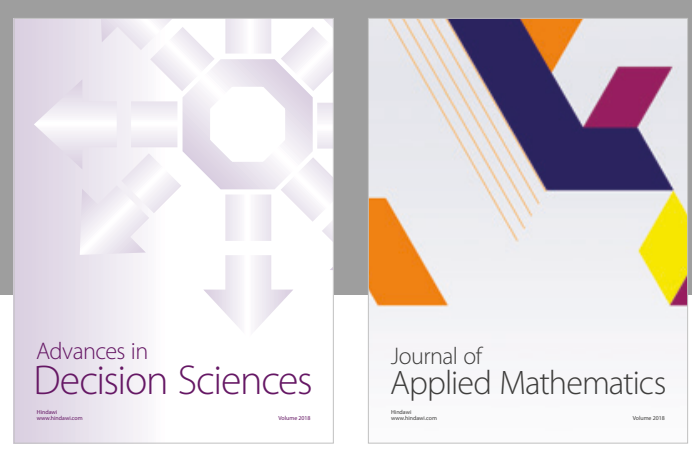

Journal of

Applied Mathematics
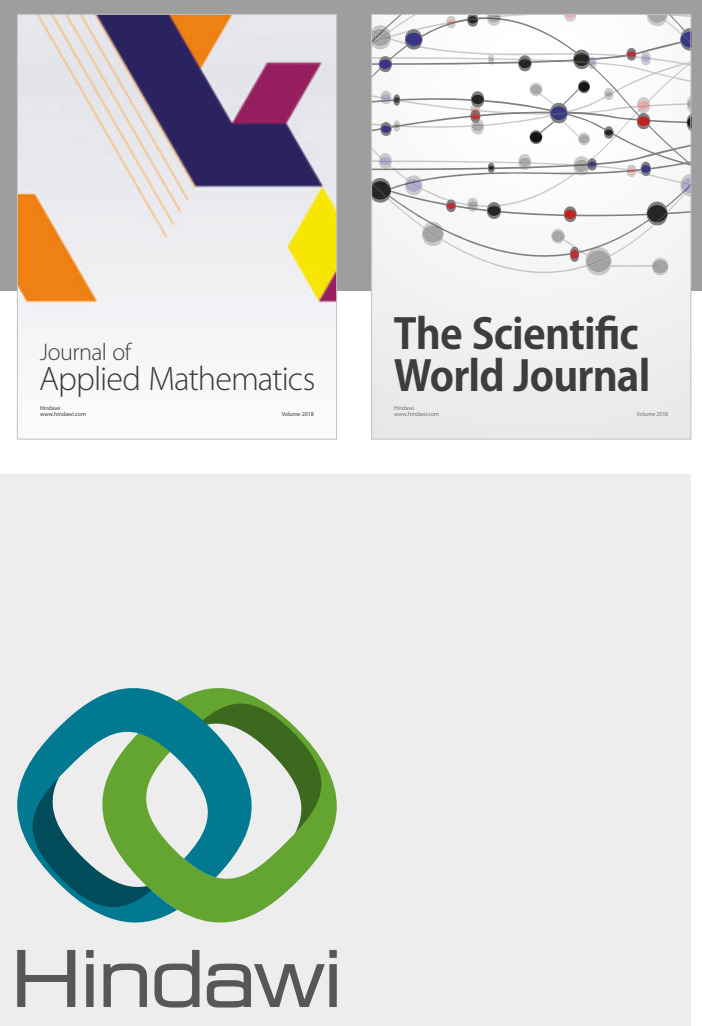

Submit your manuscripts at

www.hindawi.com

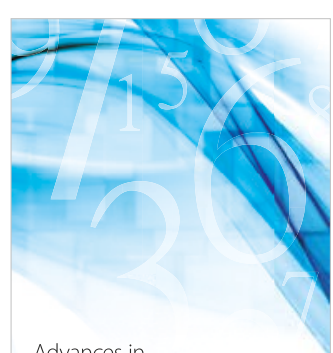

Advances in
Numerical Analysis
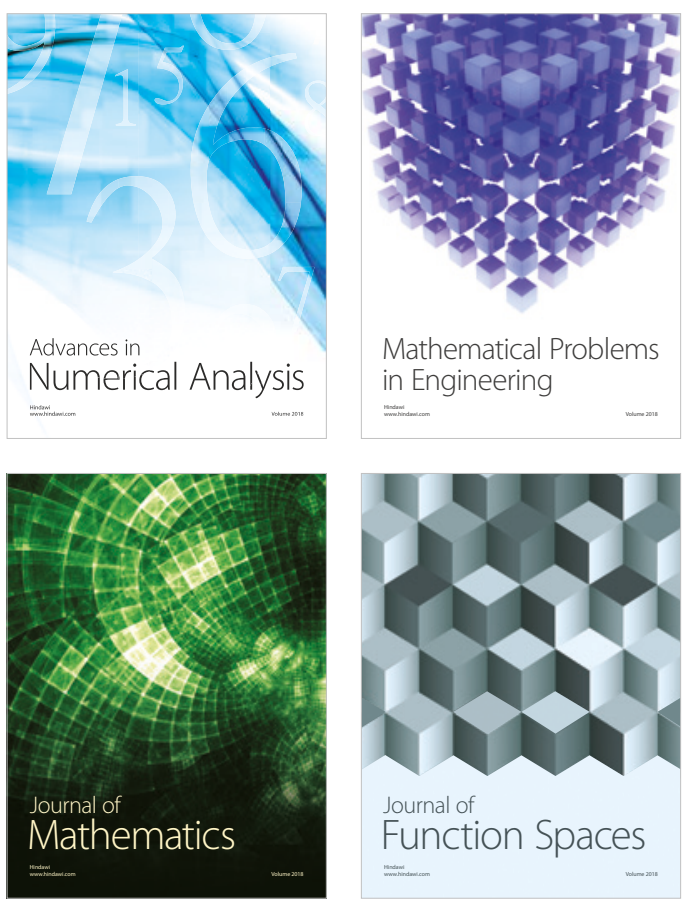

Mathematical Problems in Engineering

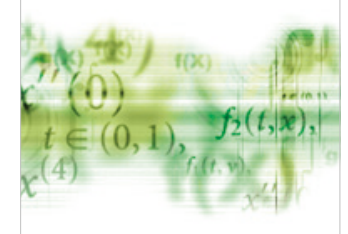

International Journal of

Differential Equations

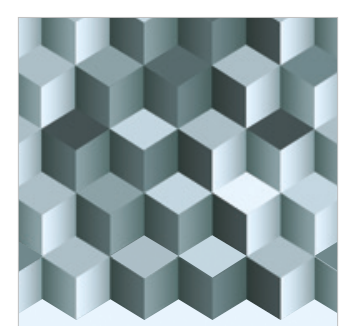

Journal of

Function Spaces



The Scientific

World Journal



Journal of

Probability and Statistics
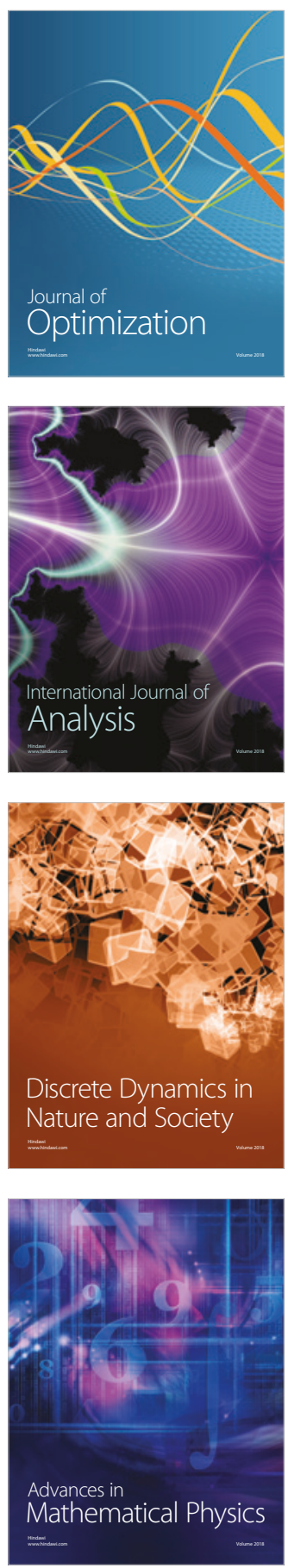